\title{
Highly Regio- and Chemoselective Palladium-Catalyzed Propargylallylation of Activated Olefins: A Novel Route to 1,7-Enyne Derivatives
}

Masilamani Jeganmohan, Muthian Shanmugasundaram, and Chien-Hong Cheng ${ }^{*}$

Department of Chemistry, Tsing Hua University, Hsinchu, Taiwan 300 ROC.

chcheng@mx.nthu.edu.tw

\section{Supporting Information}

Table of Contents

Page S2 Experimental Section

Page S2 - S11 $\quad{ }^{1} \mathrm{H}$ and ${ }^{13} \mathrm{C}$ NMR Data

Page S11 - S13 Homo decouple and NOE experimental Data.

Page S14 - S37 $\quad{ }^{1}$ H NMR Spectra 


\section{General Procedure for the Propargylallylation of Arylethylidene Malononitriles,}

\section{Allylic Chlorides and Allenylstannanes.}

A 25-mL round-bottom flask containing $\mathrm{Pd}\left(\mathrm{PPh}_{3}\right)_{4}(0.050 \mathrm{mmol})$ and arylethylidene malononitrile $(1.00 \mathrm{mmol})$ was dissolved in $3 \mathrm{ml}$ toluene and purged with nitrogen gas several times. To the flask were then added allyl chloride $(1.20 \mathrm{mmol})$ and allenylstannane $(1.20 \mathrm{mmol})$ via syringe. The reaction mixture was stirred at ambient temperature for $8 \mathrm{~h}$. The reaction mixture was then treated with $10 \mathrm{~mL}$ of saturated $\mathrm{KF}$ solution and stirred for $30 \mathrm{~min}$ at ambient temperature. The solution was filtered through a Celite and silica gel pad and washed with $50 \mathrm{~mL}$ of ether. The filtrate was washed with water, dried $\left(\mathrm{MgSO}_{4}\right)$ and concentrated in vacuo. The residue was purified on a silica gel column (hexane/EtOAc $=9 / 1)$ to give the desired product. Products $\mathbf{6}, \mathbf{8}$ and $\mathbf{1 0}$ were synthesized according to similar procedures by using substituted 2-(phenylmethylene)-1,3-indanedione substituted 2-2-dimethyl-5-(phenylmethylene)-1,3-dioxane-4,6-dione (7) and conjugated activated olefins (9) as the amphiphilic reagents, respectively.

Products 4a-m, 6a-m, 8a-d, 10a-h and 11 were obtained according to this procedure. Product yields of these reactions are listed in Tables $\mathbf{2 , 3}, \mathbf{4}$, and $\mathbf{5}$, while spectral data of these 1,7-enyne derivatives are shown below or listed in supporting information.

${ }^{1} \mathrm{H}$ NMR spectra of compounds $\mathbf{4 a - d}, \mathbf{4} \mathbf{f}-\mathbf{i}, \mathbf{4 k}, \mathbf{4 m}, \mathbf{1 0 a - c}$ and $\mathbf{1 1}$ are available in the supporting information of Org. Lett. 2003, 5, 881-884

2-(1-Phenyl-3-butynyl)-2-((E)-3-phenyl-2-propenyl)propanedinitrile (4d): pale yellow oil; ${ }^{1} \mathrm{H}$ NMR $\left(500 \mathrm{MHz}, \mathrm{CDCl}_{3}\right): \delta 7.45-7.28(\mathrm{~m}, 10 \mathrm{H}), 6.57(\mathrm{~d}, J=16.0 \mathrm{~Hz}, 1 \mathrm{H})$, 
$6.17(\mathrm{~m}, 1 \mathrm{H}), 3.31(\mathrm{dd}, J=10.5,4.5 \mathrm{~Hz}, 1 \mathrm{H}), 3.11-2.99(\mathrm{~m}, 2 \mathrm{H}), 2.69$ (dd, $J=14.0,7.5$ $\mathrm{Hz}, 1 \mathrm{H}), 2.60(\mathrm{dd}, J=14.0,7.5 \mathrm{~Hz}, 1 \mathrm{H}), 1.92(\mathrm{t}, J=3.0 \mathrm{~Hz}, 1 \mathrm{H}) ;{ }^{13} \mathrm{C} \mathrm{NMR}(125 \mathrm{MHz}$, $\left.\mathrm{CDCl}_{3}\right): \delta 138.0,135.7,134.6,129.3,129.2,128.8,128.7,128.5,126.7,118.9,114.9$, 113.9, 79.3, 71.7, 50.0, 42.9, 40.1, 22.5; HRMS calcd for $\mathrm{C}_{22} \mathrm{H}_{18} \mathrm{~N}_{2} 310.1470$, found 310.1460.

2-(2-Cyclohexenylmethyl)-2-(1-phenyl-3-butynyl)propanedinitrile (4e): pale yellow oil; ${ }^{1} \mathrm{H}$ NMR (500 MHz, $\left.\mathrm{CDCl}_{3}\right): \delta 7.46-7.38(\mathrm{~m}, 5 \mathrm{H}), 6.08$ - $6.06(\mathrm{~d}, J=5.5 \mathrm{~Hz}, 1 \mathrm{H})$, $5.73(\mathrm{~d}, J=11 \mathrm{~Hz}, 1 \mathrm{H}), 3.50-3.37(\mathrm{~m}, 1 \mathrm{H}), 3.04-2.98(\mathrm{~m}, 2 \mathrm{H}), 2.45-2.43(\mathrm{~m}, 2 \mathrm{H}), 2.09$ - $2.02(\mathrm{~m}, 4 \mathrm{H}), 1.91-1.87(\mathrm{~m}, 2 \mathrm{H}), 1.76-1.42(\mathrm{~m}, 3 \mathrm{H}) ;{ }^{13} \mathrm{C} \mathrm{NMR}\left(125 \mathrm{MHz}, \mathrm{CDCl}_{3}\right): \delta$ 134.8, 134.7, 134.2, 129.1, 129.0, 128.8, 128.6, 123.1, 121.5, 114.5, 114.3, 114.2, 113.6, $79.5,71.4,48.1,47.9,47.2,47.2,40.2,39.8,26.3,24.8,24.7,24.5,23.2,22.4,20.9$; HRMS calcd for $\mathrm{C}_{19} \mathrm{H}_{18} \mathrm{~N}_{2} 274.1470$, found 274.1476. (1:1 diastereomers).

2-(1-(4-Chlorophenyl)-3-butynyl)-2-(2-methyl-2-propenyl)propanedinitrile (4f): pale yellow oil; ${ }^{1} \mathrm{H}$ NMR (500 MHz, $\left.\mathrm{CDCl}_{3}\right): \delta 7.40(\mathrm{~d}, J=7.5 \mathrm{~Hz}, 2 \mathrm{H}), 7.32(\mathrm{~d}, J=7.5$ $\mathrm{Hz}, 2 \mathrm{H}), 5.13(\mathrm{~s}, 1 \mathrm{H}), 5.02(\mathrm{~s}, 1 \mathrm{H}), 3.27(\mathrm{dd}, J=11.0,4.5 \mathrm{~Hz}, 1 \mathrm{H}), 3.09-2.93(\mathrm{~m}, 2 \mathrm{H})$, 2.50 (d, $J=14.5 \mathrm{~Hz}, 1 \mathrm{H}), 2.31(\mathrm{~d}, J=14.5 \mathrm{~Hz}, 1 \mathrm{H}), 1.92(\mathrm{t}, J=2.5 \mathrm{~Hz}, 1 \mathrm{H}), 1.89$ (s, 3H);

${ }^{13} \mathrm{C}$ NMR (125 MHz, $\left.\mathrm{CDCl}_{3}\right): \delta 136.9,135.4,133.1,130.2,129.3,118.9,114.8,114.1$, 78.9, 71.9, 50.7, 44.1, 41.7, 23.0, 22.1; HRMS calcd for $\mathrm{C}_{17} \mathrm{H}_{15} \mathrm{ClN}_{2} 282.0924$, found 282.0917.

2-(1-(4-(Methyloxy)phenyl)-3-butynyl)-2-(2-methyl-2-propenyl)propanedini-tril e (4g): pale yellow oil; ${ }^{1} \mathrm{H}$ NMR $\left(500 \mathrm{MHz}, \mathrm{CDCl}_{3}\right): \delta 7.30(\mathrm{~d}, J=7.5 \mathrm{~Hz}, 2 \mathrm{H}), 6.92(\mathrm{~d}, J$ $=7.5 \mathrm{~Hz}, 2 \mathrm{H}), 5.11(\mathrm{~s}, 1 \mathrm{H}), 5.01(\mathrm{~s}, 1 \mathrm{H}), 3.81(\mathrm{~s}, 3 \mathrm{H}), 3.24(\mathrm{dd}, J=11.5,5.0 \mathrm{~Hz}, 1 \mathrm{H})$, $3.09-3.00(\mathrm{~m}, 2 \mathrm{H}), 2.50(\mathrm{~d}, J=14.5 \mathrm{~Hz}, 1 \mathrm{H}), 2.31(\mathrm{~d}, J=14.5 \mathrm{~Hz}, 1 \mathrm{H}), 1.86(\mathrm{t}, J=3.0$ 
$\mathrm{Hz}, 1 \mathrm{H}), 1.85(\mathrm{~s}, 3 \mathrm{H}) ;{ }^{13} \mathrm{C}$ NMR $\left(125 \mathrm{MHz}, \mathrm{CDCl}_{3}\right): \delta 160.1,137.2,129.9,126.4,118.6$, 115.2, 114.4, 114.3, 79.5, 71.4, 55.3, 50.9, 44.2, 42.1, 23.1, 22.4; HRMS calcd for $\mathrm{C}_{18} \mathrm{H}_{18} \mathrm{~N}_{2} \mathrm{O} 278.1419$, found 278.1420.

2-(2-Methyl-2-propenyl)-2-(1-(4-nitrophenyl)-3-butynyl)propanedinitrile (4h): pale yellow oil; ${ }^{1} \mathrm{H}$ NMR $\left(500 \mathrm{MHz}, \mathrm{CDCl}_{3}\right): \delta 8.29(\mathrm{~d}, J=7.5 \mathrm{~Hz}, 2 \mathrm{H}), 7.60(\mathrm{~d}, J=7.5$ $\mathrm{Hz}, 2 \mathrm{H}), 5.16(\mathrm{~s}, 1 \mathrm{H}), 5.05(\mathrm{~s}, 1 \mathrm{H}), 3.42(\mathrm{dd}, J=11.5,4.5 \mathrm{~Hz}, \mathrm{H}), 3.13-2.98(\mathrm{~m}, 2 \mathrm{H})$, $2.59(\mathrm{~d}, J=14.0 \mathrm{~Hz}, 1 \mathrm{H}), 2.35(\mathrm{~d}, J=14.5 \mathrm{~Hz}, 1 \mathrm{H}), 1.93(\mathrm{t}, J=3.0 \mathrm{~Hz}, 1 \mathrm{H}), 1.91(\mathrm{~s}, 3 \mathrm{H})$; ${ }^{13} \mathrm{C}$ NMR $\left(125 \mathrm{MHz}, \mathrm{CDCl}_{3}\right): \delta 148.5,141.9,136.5,130.0,124.2,119.3,114.3,113.8$, 78.3, 72.6, 50.8, 44.1, 41.5, 23.0, 21.9; HRMS calcd for $\mathrm{C}_{17} \mathrm{H}_{15} \mathrm{~N}_{3} 0_{2} 293.1164$, found 293.1153.

2-(2-Methyl-2-propenyl)-2-(1-(1-naphthyl)-3-butynyl)propanedinitrile (4i): pale yellow oil; ${ }^{1} \mathrm{H}$ NMR (500 MHz, $\left.\mathrm{CDCl}_{3}\right): \delta 7.91-7.84(\mathrm{~m}, 4 \mathrm{H}), 7.54-7.50(\mathrm{~m}, 3 \mathrm{H}), 5.12$ (s, 1H), $5.01(\mathrm{~s}, 1 \mathrm{H}), 3.47$ (dd, $J=10.0,5.0 \mathrm{~Hz}, 1 \mathrm{H}), 3.18-3.14(\mathrm{~m}, 2 \mathrm{H}), 2.54(\mathrm{~d}, J=$ $14.5 \mathrm{~Hz}, 1 \mathrm{H}), 2.31(\mathrm{~d}, J=14.5 \mathrm{~Hz}, 1 \mathrm{H}), 1.87(\mathrm{~s}, 3 \mathrm{H}), 1.86(\mathrm{t}, J=2.5 \mathrm{~Hz}, 1 \mathrm{H}) ;{ }^{13} \mathrm{C} \mathrm{NMR}$ (125 MHz, $\left.\mathrm{CDCl}_{3}\right): \delta 137.1,133.5,133.1,131.9,129.1,128.9,128.2,127.8,126.9,126.8$, 125.5, 118.7, 115.2, 114.3, 79.3, 71.7, 51.7, 44.3, 41.8, 23.1, 22.5; HRMS calcd for $\mathrm{C}_{21} \mathrm{H}_{18} \mathrm{~N}_{2} 298.1470$, found 298.1473.

2-Allyl-2-[1-(2-furyl)-3-butynyl]malononitrile (4j): pale yellow oil; ${ }^{1} \mathrm{H} N \mathrm{NR}$ $\left(500 \mathrm{MHz}, \mathrm{CDCl}_{3}\right): \delta 7.45(\mathrm{~d}, J=2.0 \mathrm{~Hz}, 1 \mathrm{H}), 6.41(\mathrm{~d}, J=3.5 \mathrm{~Hz}, 1 \mathrm{H}), 6.38(\mathrm{~d}, J=3.0$ Hz, 1H), $5.89-5.82(\mathrm{~m}, 1 \mathrm{H}), 5.42-5.35(\mathrm{~m}, 2 \mathrm{H}), 3.50(\mathrm{dd}, J=10.5,4.5 \mathrm{~Hz}, 1 \mathrm{H}), 3.89-$ $2.79(\mathrm{~m}, 2 \mathrm{H}), 2.58-2.47(\mathrm{~m}, 2 \mathrm{H}), 1.99(\mathrm{t}, J=3.0 \mathrm{~Hz}, 1 \mathrm{H}) ;{ }^{13} \mathrm{C} \mathrm{NMR}\left(125 \mathrm{MHz}, \mathrm{CDCl}_{3}\right)$ : $\delta 147.8,143.5,128.2,123.7,114.3,113.4,110.9,110.7,78.4,71.7,45.3,43.3,41.8,40.1$, 19.7; HRMS calcd for $\mathrm{C}_{14} \mathrm{H}_{12} \mathrm{~N}_{2} \mathrm{O} 224.0950$, found 224.0947. 
2-(1-(2-Furanyl)-3-butynyl)-2-(2-methyl-2-propenyl)propanedinitrile (4k): pale yellow oil; ${ }^{1} \mathrm{H}$ NMR $\left(500 \mathrm{MHz}, \mathrm{CDCl}_{3}\right): \delta 7.45(\mathrm{~d}, J=2.0 \mathrm{~Hz}, 1 \mathrm{H}), 6.50(\mathrm{dd}, J=5.5,3.5$ $\mathrm{Hz}, 1 \mathrm{H}), 6.41(\mathrm{~d}, J=3.0 \mathrm{~Hz}, 1 \mathrm{H}), 5.13(\mathrm{~s}, 1 \mathrm{H}), 5.04(\mathrm{~s}, 1 \mathrm{H}), 3.50(\mathrm{t}, J=4.5 \mathrm{~Hz}, 1 \mathrm{H}), 3.00$ - $2.98(\mathrm{~m}, 2 \mathrm{H}), 2.50(\mathrm{~d}, J=14.5 \mathrm{~Hz}, 1 \mathrm{H}), 2.36(\mathrm{~d}, J=14.5 \mathrm{~Hz}, 1 \mathrm{H}), 1.99(\mathrm{t}, J=3.0 \mathrm{~Hz}$, 1H), $1.90(\mathrm{~s}, 3 \mathrm{H}) ;{ }^{13} \mathrm{C} \mathrm{NMR}\left(125 \mathrm{MHz}, \mathrm{CDCl}_{3}\right): \delta 147.9,143.4,136.9,118.7,114.6$, 113.8, 110.8, 110.6, 78.7, 71.6, 45.3, 43.4, 41.1, 22.9, 21.0; HRMS calcd for $\mathrm{C}_{15} \mathrm{H}_{14} \mathrm{~N}_{2} \mathrm{O}$ 238.1106, found 238.1107.

2-Allyl-2-[1-(2-thienyl)-3-butynyl]malononitrile (4l): pale yellow oil; ${ }^{1} \mathrm{H}$ NMR (500 MHz, $\left.\mathrm{CDCl}_{3}\right): \delta 7.36(\mathrm{~d}, J=4.5 \mathrm{~Hz}, 1 \mathrm{H}), 7.19(\mathrm{~d}, J=3.5 \mathrm{~Hz}, 1 \mathrm{H}), 7.06(\mathrm{dd}, J=5.0$, $3.5 \mathrm{~Hz}, 1 \mathrm{H}), 5.90-5.83(\mathrm{~m}, 1 \mathrm{H}), 5.43-5.34(\mathrm{~m}, 2 \mathrm{H}), 3.59(\mathrm{dd}, J=11.0,4.0 \mathrm{~Hz}, 1 \mathrm{H})$, $3.10-2.92(\mathrm{~m}, 2 \mathrm{H}), 2.60-2.54(\mathrm{~m}, 2 \mathrm{H}), 1.99(\mathrm{t}, J=3.0 \mathrm{~Hz}, 1 \mathrm{H}) ;{ }^{13} \mathrm{C} \mathrm{NMR}(125 \mathrm{MHz}$, $\left.\mathrm{CDCl}_{3}\right): \delta 136.0,127.9,127.7,127.1,126.1,123.5,114.2,113.4,78.5,71.8,45.4,42.7$, 40.1, 24.0; HRMS calcd for $\mathrm{C}_{14} \mathrm{H}_{12} \mathrm{~N}_{2} \mathrm{~S} 240.0721$, found 240.0727 .

\section{2-(2-Methyl-2-propenyl)-2-(1-(2-thiophenyl)-3-butynyl)propanedinitrile (4m):} pale yellow oil; ${ }^{1} \mathrm{H}$ NMR $\left(500 \mathrm{MHz}, \mathrm{CDCl}_{3}\right): \delta 7.35(\mathrm{~d}, J=4.5 \mathrm{~Hz}, 1 \mathrm{H}), 7.19(\mathrm{~d}, J=3.5$ $\mathrm{Hz}, 1 \mathrm{H}), 7.06$ (dd, $J=5.0,3.5 \mathrm{~Hz}, 1 \mathrm{H}), 5.14$ (s, 1H), 5.05 (s, 1H), 3.60 (dd, $J=11.0,4.0$ Hz, 1H), 3.14 - 2.94 (m, 2H), $2.52(\mathrm{~d}, J=14.0 \mathrm{~Hz}, 1 \mathrm{H}), 2.42(\mathrm{~d}, J=14.0 \mathrm{~Hz}, 1 \mathrm{H}), 1.97$ (t, $J=3.0 \mathrm{~Hz}, 1 \mathrm{H}), 1.90(\mathrm{~s}, 3 \mathrm{H}) ;{ }^{13} \mathrm{C} \mathrm{NMR}\left(125 \mathrm{MHz}, \mathrm{CDCl}_{3}\right): \delta 137.0,136.5,128.1,127.4$, 126.4, 118.8, 114.9, 114.1, 78.8, 71.9, 47.3, 43.9, 42.2, 24.2, 23.1; HRMS calcd for $\mathrm{C}_{15} \mathrm{H}_{14} \mathrm{~N}_{2} \mathrm{~S} 254.0878$, found 254.0886

2-(2-Cyclohexenyl)-2-(1-phenyl-3-butynyl)-1,3-indanedione (6d): pale yellow oil; ${ }^{1} \mathrm{H}$ NMR (600 MHz, $\left.\mathrm{CDCl}_{3}\right): \delta 7.90-7.57(\mathrm{~m}, 4 \mathrm{H}), 7.03-6.95(\mathrm{~m}, 4 \mathrm{H}), 6.09-5.89(\mathrm{~m}$, 
$1 \mathrm{H}), 5.55-4.97(\mathrm{~m}, 1 \mathrm{H}), 3.77(\mathrm{dd}, J=12,4.2 \mathrm{~Hz}, 1 \mathrm{H}), 3.53(\mathrm{dd}, J=9,6.6 \mathrm{~Hz}, 1 \mathrm{H})$, $3.19-2.87(\mathrm{~m}, 2 \mathrm{H}), 2.01-1.71(\mathrm{~m}, 9 \mathrm{H}) ;{ }^{13} \mathrm{C} \mathrm{NMR}\left(125 \mathrm{MHz}, \mathrm{CDCl}_{3}\right): \delta 204.9,204.5$, 202.6, 202.3, 142.6, 142.5, 137.8, 135.3, 135.1, 130.9, 130.3, 129.3, 129.3, 127.9, 127.9, $127.1,127.0,125.5,124.8,122.5,122.3,122.1,122.0,82.3,69.9,63.2,62.4,46.5,46.1$, 39.5, 38.2, 26.3, 24.9, 23.3, 22.2,19.2, 18.6, 14.1; HRMS calcd for $\mathrm{C}_{25} \mathrm{H}_{22} \mathrm{O}_{2} 354.1620$, found 354.1616. (1:1 diastereomers).

\section{2-[1-(4-Chlorophenyl)-3-butynyl]-2-(2-methylallyl)-1,3-indanedione}

(6e):

viscous oil; ${ }^{1} \mathrm{H}$ NMR $\left(500 \mathrm{MHz}, \mathrm{CDCl}_{3}\right): \delta 7.80(\mathrm{~d}, J=7.2,1 \mathrm{H}), 7.69-7.61(\mathrm{~m}, 3 \mathrm{H})$, $7.05-7.01(\mathrm{~m}, 5 \mathrm{H}), 4.53(\mathrm{~s}, 2 \mathrm{H}), 3.42(\mathrm{dd}, J=9.0,7.5 \mathrm{~Hz}, 1 \mathrm{H}), 2.83-2.81(\mathrm{~m}, 2 \mathrm{H})$, $2.75(\mathrm{~d}, J=13.5 \mathrm{~Hz}, 1 \mathrm{H}), 2.49(\mathrm{~d}, J=13.5 \mathrm{~Hz}, 1 \mathrm{H}), 1.72(\mathrm{t}, J=2.5 \mathrm{~Hz}, 1 \mathrm{H}), 1.31(\mathrm{~s}, 3 \mathrm{H})$; ${ }^{13} \mathrm{C}$ NMR $\left(125 \mathrm{MHz}, \mathrm{CDCl}_{3}\right): \delta 203.5,202.5,142.8,142.5,139.4,136.7,135.6,135.6$, 133.0, 130.6, 128.1, 122.7, 122.5, 116.9, 81.7, 70.5, 60.9, 49.8, 42.1, 23.9, 20.1; HRMS calcd for $\mathrm{C}_{23} \mathrm{H}_{19} \mathrm{ClO}_{2} 362.1074$, found 362.1070 .

2-[1-(4-Bromophenyl)-3-butynyl]-2-(2-methylallyl)-1,3-indanedione

(6f):

viscous oil; ${ }^{1} \mathrm{H}$ NMR $\left(600 \mathrm{MHz}, \mathrm{CDCl}_{3}\right): \delta 7.81-7.66(\mathrm{~m}, 4 \mathrm{H}), 7.19(\mathrm{~d}, J=8.4 \mathrm{~Hz}, 2 \mathrm{H})$, $6.97(\mathrm{~d}, J=8.4 \mathrm{~Hz}, 2 \mathrm{H}) 4.53(\mathrm{~s}, 2 \mathrm{H}), 3.40(\mathrm{dd}, J=9.0,6.6 \mathrm{~Hz}, 1 \mathrm{H}), 2.82-2.80(\mathrm{~m}, 2 \mathrm{H})$, $2.74(\mathrm{~d}, J=13.5 \mathrm{~Hz}, 1 \mathrm{H}), 2.48(\mathrm{~d}, J=13.0 \mathrm{~Hz}, 1 \mathrm{H}), 1.71(\mathrm{t}, J=2.4 \mathrm{~Hz}, 1 \mathrm{H}), 1.30(\mathrm{~s}, 3 \mathrm{H})$; ${ }^{13} \mathrm{C}$ NMR $\left(125 \mathrm{MHz}, \mathrm{CDCl}_{3}\right): \delta 203.5,202.4,142.8,142.5,139.4,137.2,135.6,135.6$, 131.1, 127.8, 122.7, 121.3, 116.9, 81.7, 70.5, 60.8, 49.8, 42.1, 23.9, 20.2; HRMS calcd for $\mathrm{C}_{23} \mathrm{H}_{19} \mathrm{BrO}_{2} 406.0568$, found 406.0570 .

2-[1-(4-Methoxyphenyl)-3-butynyl]-2-(2-methylallyl)-1,3-indanedione

$(6 g)$ :

viscous oil; ${ }^{1} \mathrm{H}$ NMR $\left(500 \mathrm{MHz}, \mathrm{CDCl}_{3}\right): \delta 7.79(\mathrm{~d}, J=7.2 \mathrm{~Hz}, 1 \mathrm{H}) 7.70-7.62(\mathrm{~m}, 3 \mathrm{H})$, 
$6.98(\mathrm{~d}, J=8.4 \mathrm{~Hz}, 2 \mathrm{H}), 6.59$ (d, $J=8.4 \mathrm{~Hz}, 2 \mathrm{H}), 4.53$ (s, 2H), 3.63 (s, 3H), 3.39 (dd, $J=$ 9.5, $7.0 \mathrm{~Hz}, 1 \mathrm{H}), 2.85-2.82(\mathrm{~m}, 2 \mathrm{H}), 2.79(\mathrm{~d}, J=13.0 \mathrm{~Hz}, 1 \mathrm{H}), 2.52(\mathrm{~d}, J=13.0 \mathrm{~Hz}$, $1 \mathrm{H}), 1.72(\mathrm{t}, J=2.5 \mathrm{~Hz}, 1 \mathrm{H}), 1.33(\mathrm{~s}, 3 \mathrm{H}) ;{ }^{13} \mathrm{C} \mathrm{NMR}\left(125 \mathrm{MHz}, \mathrm{CDCl}_{3}\right): \delta 203.9,202.8$, $158.5,142.9,142.7,139.8,135.4,135.3,130.2,122.6,122.5,116.7,113.3,82.3,70.1$, 61.3, 54.9, 49.8, 41.9, 24.0, 20.2; HRMS calcd for $\mathrm{C}_{24} \mathrm{H}_{22} \mathrm{O}_{3} 358.1569$, found 358.1571.

2-(2-Methylallyl)-2-[1-(4-nitrophenyl)-3-butynyl]-1,3-indanedione (6h): viscous oil; ${ }^{1} \mathrm{H}$ NMR (500 MHz, $\left.\mathrm{CDCl}_{3}\right): \delta 7.95(\mathrm{~d}, J=8.5 \mathrm{~Hz}, 2 \mathrm{H}), 7.82(\mathrm{~d}, J=7.5 \mathrm{~Hz}, 1 \mathrm{H})$, $7.73-7.67$ (m, 3H), $7.30(\mathrm{~d}, J=9.0 \mathrm{~Hz}, 2 \mathrm{H}), 4.54(\mathrm{~s}, 2 \mathrm{H}), 3.57$ (dd, $J=9.5,7.5 \mathrm{~Hz}, 1 \mathrm{H})$, $2.89-2.80(\mathrm{~m}, 2 \mathrm{H}), 2.75(\mathrm{~d}, J=13.0 \mathrm{~Hz}, 1 \mathrm{H}), 2.47(\mathrm{~d}, J=13.0 \mathrm{~Hz}, 1 \mathrm{H}), 1.70(\mathrm{t}, J=2.5$ $\mathrm{Hz}, 1 \mathrm{H}), 1.33(\mathrm{~s}, 3 \mathrm{H}) ;{ }^{13} \mathrm{C} \mathrm{NMR}\left(125 \mathrm{MHz}, \mathrm{CDCl}_{3}\right): \delta 203.0,202.1,147.0,145.8,142.6$, $142.4,139.0,135.9,135.9,130.3,123.1,122.9,122.7,117.4,81.0,70.9,60.6,49.9,42.2$ 23.9,19.9; HRMS calcd for $\mathrm{C}_{23} \mathrm{H}_{19} \mathrm{NO}_{4}$ 373.1311, found 373.1311.

\section{4-1-[2-(2-Methylallyl)-1,3-dioxo-2,3-dihydro-1H-2-indenyl]-3-butynylbenzonitri}

le (6i): viscous oil; ${ }^{1} \mathrm{H}$ NMR $\left(600 \mathrm{MHz}, \mathrm{CDCl}_{3}\right): \delta 7.81-7.67(\mathrm{~m}, 4 \mathrm{H}), 7.36(\mathrm{~d}, J=8.4$ Hz, 2H), $7.21(\mathrm{~d}, J=8.4 \mathrm{~Hz}, 2 \mathrm{H}), 4.53-4.52(\mathrm{~m}, 2 \mathrm{H}), 3.49(\mathrm{dd}, J=10.6,5.4 \mathrm{~Hz}, 1 \mathrm{H})$, $2.86-2.81(\mathrm{~m}, 2 \mathrm{H}), 2.73(\mathrm{~d}, J=12.6 \mathrm{~Hz}, 1 \mathrm{H}), 2.45(\mathrm{~d}, J=12.6 \mathrm{~Hz}, 1 \mathrm{H}), 1.70(\mathrm{t}, J=2.4$ $\mathrm{Hz}, 1 \mathrm{H}), 1.29$ (s, 3H); ${ }^{13} \mathrm{C}$ NMR (125 MHz, $\left.\mathrm{CDCl}_{3}\right): \delta 202.9,202.9,143.7,142.6,142.3$, 139.1, 135.9, 135.8, 131.7, 130.1, 122.8, 122.6, 118.4, 117.2, 111.1, 81.1, 70.8, 60.6, 50.2, 42.0, 23.9,19.7; HRMS calcd for $\mathrm{C}_{24} \mathrm{H}_{19} \mathrm{NO}_{2} 353.1416$, found 353.1415 .

2-(2-Methylallyl)-2-[1-(4-biphenyl)-3-butynyl]-1,3-indanedione (6j): viscous oil; ${ }^{1} \mathrm{H}$ NMR $\left(500 \mathrm{MHz}, \mathrm{CDCl}_{3}\right): \delta 7.82(\mathrm{~d}, J=8.0 \mathrm{~Hz}, 1 \mathrm{H}), 7.65-7.60(\mathrm{~m}, 3 \mathrm{H}), 7.43(\mathrm{~d}, J=$ 
$8.5 \mathrm{~Hz}, 2 \mathrm{H}), 7.36-7.25(\mathrm{~m}, 5 \mathrm{H}), 7.15(\mathrm{~d}, J=8.5,2 \mathrm{H}), 4.56(\mathrm{~d}, J=8.0 \mathrm{~Hz}, 2 \mathrm{H}), 3.50(\mathrm{t}, J$ $=8.0 \mathrm{~Hz}, 1 \mathrm{H}), 2.92-2.90(\mathrm{dd}, J=7.5,2.5 \mathrm{~Hz} 2 \mathrm{H}), 2.84(\mathrm{~d}, J=13.5 \mathrm{~Hz}, 1 \mathrm{H}), 2.57(\mathrm{~d}, J=$ $13.0 \mathrm{~Hz}, 1 \mathrm{H}), 1.76(\mathrm{t}, J=2.5 \mathrm{~Hz}, 1 \mathrm{H}), 1.36(\mathrm{~s}, 3 \mathrm{H}) ;{ }^{13} \mathrm{C}$ NMR $\left(125 \mathrm{MHz}, \mathrm{CDCl}_{3}\right): \delta$ 203.7, 202.7, 142.9, 142.7, 140.4, 139.8, 139.7, 137.3, 135.4, 135.4, 129.6, 128.6, 127.2, $126.8,126.5,122.7,122.4,116.8,82.2,70.3,61.2,50.3,41.9,24.0,20.1 ;$ HRMS calcd for $\mathrm{C}_{29} \mathrm{H}_{24} \mathrm{O}_{2} 404.1776$, found 404.1778 .

2-(2-Methylallyl)-2-[1-(1-naphthyl)-3-butynyl]-1,3-indanedione (6k): viscous oil; ${ }^{1} \mathrm{H}$ NMR (500 MHz, $\left.\mathrm{CDCl}_{3}\right): \delta 8.33(\mathrm{~d}, J=9.0 \mathrm{~Hz}, 1 \mathrm{H}), 7.87(\mathrm{~d}, J=8.0 \mathrm{~Hz}, 1 \mathrm{H}), 7.73-$ $7.45(\mathrm{~m}, 8 \mathrm{H}), 7.21(\mathrm{t}, J=6 \mathrm{~Hz}, 1 \mathrm{H}), 4.60(\mathrm{dd}, J=9.5,6.0 \mathrm{~Hz}, 1 \mathrm{H}), 4.50(\mathrm{~d}, J=7.5, \mathrm{~Hz}$, 2H), $2.99-2.83(\mathrm{~m}, 2 \mathrm{H}), 2.81(\mathrm{~d}, J=13.0 \mathrm{~Hz}, 1 \mathrm{H}), 2.53(\mathrm{~d}, J=13.5 \mathrm{~Hz}, 1 \mathrm{H}), 1.54(\mathrm{t}, J=$ $2.5 \mathrm{~Hz}, 1 \mathrm{H}), 1.30(\mathrm{~s}, 3 \mathrm{H}) ;{ }^{13} \mathrm{C} \mathrm{NMR}\left(125 \mathrm{MHz}, \mathrm{CDCl}_{3}\right): \delta 204.2,202.2,143.2,142.7$, $139.6,135.3,135.3,133.6,132.7,128.6,127.8,126.1,125.9,125.4,124.9,124.7,123.8$, 122.7, 122.4, 116.8, 82.0, 70.2, 61.2, 42.4, 34.0, 22.6, 14.2; HRMS calcd for $\mathrm{C}_{27} \mathrm{H}_{22} \mathrm{O}_{2}$ 378.1620 , found 378.1622 .

2-(2-Methylallyl)-2-[1-(2-thienyl)-3-butynyl]-1,3-indanedione (61): viscous oil; ${ }^{1} \mathrm{H}$ NMR $\left(600 \mathrm{MHz}, \mathrm{CDCl}_{3}\right): \delta 7.83-7.67(\mathrm{~m}, 4 \mathrm{H}), 6.94(\mathrm{dd}, J=4.2,3.6 \mathrm{~Hz}, 1 \mathrm{H}), 6.78-$ $6.77(\mathrm{dd}, J=3.6,1.2 \mathrm{~Hz}, 1 \mathrm{H}), 6.69(\mathrm{dd}, J=5.4,3.6 \mathrm{~Hz}, 1 \mathrm{H}), 4.54-4.52(\mathrm{~m}, 1 \mathrm{H}), 3.75$ $(\mathrm{dd}, J=10.2,5.4 \mathrm{~Hz}, 1 \mathrm{H}), 2.85-2.77(\mathrm{~m}, 3 \mathrm{H}), 2.56(\mathrm{~d}, J=13.0 \mathrm{~Hz}, 1 \mathrm{H}), 1.78(\mathrm{t}, J=2.4$ $\mathrm{Hz}, 1 \mathrm{H}), 1.32$ (s, 3H); ${ }^{13} \mathrm{C} \mathrm{NMR}\left(125 \mathrm{MHz}, \mathrm{CDCl}_{3}\right): \delta 203.3,202.4,143.0,142.6,140.9$, $139.5,135.4,127.7,126.1,124.5,122.7,122.5,116.9,81.6,70.4,61.1,45.8,41.9,23.9$, 21.9; HRMS calcd for $\mathrm{C}_{21} \mathrm{H}_{18} \mathrm{O}_{2} \mathrm{~S} 334.1027$, found 334.1029. 
viscous oil; ${ }^{1} \mathrm{H}$ NMR (500 MHz, $\left.\mathrm{CDCl}_{3}\right): \delta 7.85(\mathrm{~d}, J=8.0 \mathrm{~Hz}, 1 \mathrm{H}), 7.68(\mathrm{~d}, J=7.5 \mathrm{~Hz}$, 1H), $7.62-7.52(\mathrm{~m}, 2 \mathrm{H}), 7.33-7.31(\mathrm{~m}, 1 \mathrm{H}), 7.11-7.01(\mathrm{~m}, 3 \mathrm{H}), 6.47(\mathrm{~s}, 1 \mathrm{H}), 4.58(\mathrm{~s}$, 2H), $3.66-3.63 .(\mathrm{dd}, J=11.0,5.0 \mathrm{~Hz}, 1 \mathrm{H}), 3.00-2.85(\mathrm{~m}, 3 \mathrm{H}), 2.62(\mathrm{~d}, J=13.0 \mathrm{~Hz}$, $1 \mathrm{H}), 1.81(\mathrm{t}, J=2.5 \mathrm{~Hz}, 1 \mathrm{H}), 1.38(\mathrm{~s}, 3 \mathrm{H}) ;{ }^{13} \mathrm{C} \mathrm{NMR}\left(125 \mathrm{MHz}, \mathrm{CDCl}_{3}\right): \delta 201.9,201.7$, $154.4,154.2,142.5,141.9,139.4,135.3,135.2,127.6,123.7,122.6,122.5,120.7,116.8$, 110.6, 106.4, 81.2, 70.5, 59.9, 44.9, 40.9, 24.0, 18.5; HRMS calcd for $\mathrm{C}_{25} \mathrm{H}_{20} \mathrm{O}_{3} 368.1412$, found 368.1413 .

\section{2,2-Dimethyl-5-[1-(4-nitrophenyl)-3-butynyl]-5-[(E)-3-phenyl-2-propenyl]-1,3-d} ioxane-4,6-dione (8c): pale yellow oil; ${ }^{1} \mathrm{H}$ NMR (600 MHz, $\left.\mathrm{CDCl}_{3}\right): \delta 8.13(\mathrm{~d}, J=8.4$ Hz, 2H), 7.34 (d, $J=8.4 \mathrm{~Hz}, 2 \mathrm{H}), 7.23-7.17$ (m, 5H), 6.48 (d, $J=15.6 \mathrm{~Hz}, 1 \mathrm{H}), 5.95-$ $5.92(\mathrm{~m}, 1 \mathrm{H}), 3.78-3.76(\mathrm{dd}, J=9.4,6.6 \mathrm{~Hz}, 1 \mathrm{H}), 3.13-3.05(\mathrm{~m}, 3 \mathrm{H}), 2.91-2.88(\mathrm{~m}$, 1H), $1.76(\mathrm{t}, J=2.4 \mathrm{~Hz}, 1 \mathrm{H}), 1.47(\mathrm{~S}, 3 \mathrm{H}), 0.78(\mathrm{~s}, 3 \mathrm{H}) ;{ }^{13} \mathrm{C} \mathrm{NMR}\left(150 \mathrm{MHz}, \mathrm{CDCl}_{3}\right): \delta$ $167.5,166.9,147.8,144.9,136.9,135.8,130.5,128.7,128.6,126.3,123.8,120.9,106.5$, 80.6, 71.2, 59.4, 51.7, 39.9, 30.2, 28.4, 19.5; HRMS calcd for $\mathrm{C}_{25} \mathrm{H}_{23} \mathrm{NO}_{6} 433.1525$, found 233.1530 .

\section{5-[1-(4-Methoxyphenyl)-3-butynyl]-2,2-dimethyl-5-(2-methylallyl)-1,3-dioxane-}

4,6-dione (8d): pale yellow oil; ${ }^{1} \mathrm{H}$ NMR (600 MHz, $\left.\mathrm{CDCl}_{3}\right)$ : $\delta 7.04(\mathrm{~d}, J=8.4 \mathrm{~Hz}, 2 \mathrm{H})$, $6.77(\mathrm{~d}, J=8.4 \mathrm{~Hz}, 2 \mathrm{H}), 4.89(\mathrm{~s}, 1 \mathrm{H}), 4.82(\mathrm{~s}, 1 \mathrm{H}), 3.70(\mathrm{~s}, 3 \mathrm{H}), 3.57(\mathrm{dd}, J=9.6,6.6 \mathrm{~Hz}$, 1H), $3.01-2.98(\mathrm{~m}, 3 \mathrm{H}), 2.70(\mathrm{~d}, J=12.6,1 \mathrm{H}), 1.74(\mathrm{t}, J=2.4 \mathrm{~Hz}, 1 \mathrm{H}), 1.66(\mathrm{~s}, 3 \mathrm{H})$, $1.43(\mathrm{~s}, 3 \mathrm{H}), 0.67(\mathrm{~s}, 3 \mathrm{H}) ;{ }^{13} \mathrm{C}$ NMR $\left(150 \mathrm{MHz}, \mathrm{CDCl}_{3}\right): \delta 167.9,167.3,159.3,139.3$, $130.3,129.8,118.3,114.1,106.1,81.9,69.9,59.5,55.2,51.4,44.2,29.9,28.3,19.6$ HRMS calcd for $\mathrm{C}_{21} \mathrm{H}_{24} \mathrm{O}_{5} 356.1624$, found 356.1626 . 


\section{2-(2-Methyl-2-propenyl)-2-(1-((E)-2-phenyl-1-ethenyl)-3-butynyl)propanedinitr}

ile (10b): pale yellow oil; ${ }^{1} \mathrm{H}$ NMR $\left(500 \mathrm{MHz}, \mathrm{CDCl}_{3}\right): \delta 7.42(\mathrm{~d}, J=7.5 \mathrm{~Hz}, 2 \mathrm{H}), 7.36$ $7.30(\mathrm{~m}, 3 \mathrm{H}), 6.69$ (d, $J=15.5 \mathrm{~Hz}, 1 \mathrm{H}), 6.04(\mathrm{dd}, J=15.5,9.5 \mathrm{~Hz}, 1 \mathrm{H}), 5.15(\mathrm{~s}, 1 \mathrm{H})$, $5.08(\mathrm{~s}, 1 \mathrm{H}), 2.93-2.84(\mathrm{~m}, 2 \mathrm{H}), 2.73-2.65(\mathrm{~m}, 2 \mathrm{H}), 2.55(\mathrm{~d}, J=14.5 \mathrm{~Hz}, 1 \mathrm{H}), 2.07(\mathrm{t}, J$ $=3.0 \mathrm{~Hz}, 1 \mathrm{H}), 1.94(\mathrm{~s}, 3 \mathrm{H}) ;{ }^{13} \mathrm{C} \mathrm{NMR}\left(125 \mathrm{MHz}, \mathrm{CDCl}_{3}\right): \delta 138.0,137.1,135.4,128.8$, $128.7,126.8,122.1,118.8,114.7,114.1,79.0,72.2,49.5,43.9,40.8,23.1,22.2$; HRMS calcd for $\mathrm{C}_{19} \mathrm{H}_{18} \mathrm{~N}_{2} 274.1470$, found 274.1477.

\section{2-(2-Methyl-2-propenyl)-2-(1-((E)-2-phenyl-1-ethenyl)-3-butynyl)propanedinitr}

ile (10c): pale yellow oil; ${ }^{1} \mathrm{H}$ NMR $\left(500 \mathrm{MHz}, \mathrm{CDCl}_{3}\right): \delta 7.38-7.26(\mathrm{~m}, 10 \mathrm{H}), 6.68(\mathrm{t}, J$ $=15.5 \mathrm{~Hz}, 2 \mathrm{H}), 6.20(\mathrm{~m}, 1 \mathrm{H}), 6.07(\mathrm{dd}, J=16.0,9.5 \mathrm{~Hz}, 1 \mathrm{H}), 2.97-2.70(\mathrm{~m}, 5 \mathrm{H}), 2.07$ $(\mathrm{t}, J=3.0 \mathrm{~Hz}, 1 \mathrm{H}) ;{ }^{13} \mathrm{C}$ NMR $\left(125 \mathrm{MHz}, \mathrm{CDCl}_{3}\right): \delta 138.1,138.0,135.6,135.4,128.8$, $128.7,128.6,127.4,126.8,126.7,121.9,118.7,114.4,113.8,78.9,72.3,48.1,41.7,39.9$, 22.3; HRMS calcd for $\mathrm{C}_{24} \mathrm{H}_{20} \mathrm{~N}_{2} 336.1626$, found 336.1626

2-(2-Methylallyl)-2-1-[(E)-2-phenyl-1-ethenyl]-3-butynyl-1,3-indanedione (10e): pale yellow oil; ${ }^{1} \mathrm{H}$ NMR (600 MHz, $\left.\mathrm{CDCl}_{3}\right): \delta 7.89-7.84(\mathrm{~m}, 2 \mathrm{H}), 7.74-7.72(\mathrm{~m}, 2 \mathrm{H})$, $7.25-7.12(\mathrm{~m}, 5 \mathrm{H}), 6.42(\mathrm{~d}, J=15.6 \mathrm{~Hz}, 1 \mathrm{H}), 6.13(\mathrm{dd}, J=10.2,5.4 \mathrm{~Hz}, 1 \mathrm{H}), 4.49(\mathrm{~s}$, 2H), $2.91-2.87(\mathrm{~m}, 2 \mathrm{H}), 2.63(\mathrm{~d}, J=13.2 \mathrm{~Hz}, 1 \mathrm{H}), 2.54(\mathrm{~d}, J=13.2 \mathrm{~Hz}, 1 \mathrm{H}), 2.28-$ $2.16(\mathrm{~m}, 2 \mathrm{H}), 1.76(\mathrm{t}, J=2.4 \mathrm{~Hz}, 1 \mathrm{H}), 1.29(\mathrm{~s}, 3 \mathrm{H}),{ }^{13} \mathrm{C} \mathrm{NMR}\left(150 \mathrm{MHz}, \mathrm{CDCl}_{3}\right): \delta$ 203.6, 202.8, 143.1, 142.9, 139.8, 136.7, 135.8, 135.6, 134.3, 128.4, 127.5, 126.8, 126.5, 122.6, 122.7, 116.6, 81.3, 71.0, 60.5, 48.6, 42.6, 24.0, 20.9; HRMS calcd for $\mathrm{C}_{24} \mathrm{H}_{20} \mathrm{O}_{2}$ 340.1463 , found 340.1460 .

2,2-Dimethyl-5-(2-methylallyl)-5-1-[(E)-2-phenyl-1-ethenyl]-3-butynyl-1,3-dioxa 
ne-4,6-dione (10g): pale yellow oil; ${ }^{1} \mathrm{H}$ NMR $\left(600 \mathrm{MHz}, \mathrm{CDCl}_{3}\right): \delta 7.33(\mathrm{~d}, J=7.8 \mathrm{~Hz}$, 2H), $7.25(\mathrm{t}, J=7.2 \mathrm{~Hz} 1 \mathrm{H}), 7.18(\mathrm{t}, J=7.2 \mathrm{~Hz} 1 \mathrm{H}), 6.47(\mathrm{~d}, J=15.6 \mathrm{~Hz}, 1 \mathrm{H}), 6.03$ (dd, $J=15.6,9.6 \mathrm{~Hz}, 2 \mathrm{H}), 5.63-5.58(\mathrm{~m}, 1 \mathrm{H}), 5.18-5.11(\mathrm{~m}, 2 \mathrm{H}), 3.08-3.06(\mathrm{~m}, 1 \mathrm{H}), 2.71$ $(\mathrm{s}, 2 \mathrm{H}), 2.60-2.56(\mathrm{~m}, 1 \mathrm{H}), 2.45-2.42(\mathrm{~m}, 1 \mathrm{H}), 1.91(\mathrm{t}, J=2.4 \mathrm{~Hz}, 1 \mathrm{H}), 1.65(\mathrm{~s}, 3 \mathrm{H})$, $1.58(\mathrm{~s}, 3 \mathrm{H}), 1.54(\mathrm{~s}, 3 \mathrm{H}) ;{ }^{13} \mathrm{C} \mathrm{NMR}\left(150 \mathrm{MHz}, \mathrm{CDCl}_{3}\right): \delta 168.1,167.4,139.6,136.1$, $135.5,128.6,127.9,126.6,125.6,118.5,106.3,80.7,71.2,57.8,51.5,43.8,30.4,29.3$, 23.9, 20.8; HRMS calcd for $\mathrm{C}_{22} \mathrm{H}_{24} \mathrm{O}_{4} 352.1675$, found 352.1670.

\section{2,2-Dimethyl-5-1-[(E)-2-phenyl-1-ethenyl]-3-butynyl-5-[(E)-3-phenyl-2-propeny}

1]-1,3-dioxane-4,6-dione (10h): pale yellow oil; ${ }^{1} \mathrm{H}$ NMR (600 MHz, $\left.\mathrm{CDCl}_{3}\right): \delta 7.35-$ $7.18(\mathrm{~m}, 10 \mathrm{H}), 6.51(\mathrm{~d}, J=15.6 \mathrm{~Hz}, 1 \mathrm{H}), 6.44(\mathrm{dd}, J=15.6,9.6 \mathrm{~Hz}, 2 \mathrm{H}), 6.06-5.98(\mathrm{~m}$, 2H), $3.13-3.12(\mathrm{~m}, 1 \mathrm{H}), 2.88(\mathrm{~d}, J=7.8 \mathrm{~Hz}, 2 \mathrm{H}), 2.64-2.60(\mathrm{~m}, 1 \mathrm{H}), 2.47-2.44(\mathrm{~m}$, 1H), $1.93(\mathrm{t}, J=2.4 \mathrm{~Hz}, 1 \mathrm{H}), 1.46(\mathrm{~s}, 3 \mathrm{H}), 1.43(\mathrm{~s}, 3 \mathrm{H}) ;{ }^{13} \mathrm{C} \mathrm{NMR}\left(150 \mathrm{MHz}, \mathrm{CDCl}_{3}\right): \delta$ $168.6,167.5,136.1,136.0,135.5,128.6,127.3,126.7,126.3,124.3,121.3,108.7,80.1$, 71.3, 56.9, 51.4, 41.8, 31.7, 29.8, 21.2; HRMS calcd for $\mathrm{C}_{27} \mathrm{H}_{26} \mathrm{O}_{4}$ 414.1831, found 414.1836.

The regiochemistry of $\mathbf{4 a , b}$ was established by ${ }^{1} \mathrm{H}-{ }^{1} \mathrm{H}$ decoupling experiments. For example, on irradiating the $\mathrm{H}_{\mathrm{a}}$ proton at $\delta 3.26$ in $\mathbf{4 a}$, the multiplet at $\delta 3.03\left(\mathrm{H}_{\mathrm{b}}\right.$ and $\mathrm{H}_{\mathrm{c}}$ protons) collapses partially. Irradiation of the two protons $\left(\mathrm{H}_{\mathrm{b}}\right.$ and $\left.\mathrm{H}_{\mathrm{c}}\right)$ multiplet at $\delta 3.03$ causes the signal at $\delta 3.26$ (doublet of a doublet) to a sharp singlet and also converts the triplet at $\delta 1.90\left(\mathrm{H}_{\mathrm{d}}\right.$ proton) to a sharp singlet. Irradiation of the $\mathrm{H}_{\mathrm{d}}$ proton at $\delta 1.90$ leads to partial collapse of the multiplet at $\delta 3.03\left(\mathrm{H}_{\mathrm{b}}\right.$ and $\mathrm{H}_{\mathrm{c}}$ protons).

Similarly, irradiation of the $\mathrm{H}_{\mathrm{a}}$ proton at $\delta 3.28$ in $\mathbf{4 b}$ led to partial collapse of the 
multiplet at $\delta 3.05\left(\mathrm{H}_{\mathrm{b}}\right.$ and $\mathrm{H}_{\mathrm{c}}$ protons $)$. Irradiation of the two protons $\left(\mathrm{H}_{\mathrm{b}}\right.$ and $\left.\mathrm{H}_{\mathrm{c}}\right)$ multiplet at $\delta 3.05$ converts the doublet of a doublet at $\delta 3.28$ to a sharp singlet and also the triplet at $\delta 1.90\left(\mathrm{H}_{\mathrm{d}}\right.$ proton) to a sharp singlet. Irradiation of the $\mathrm{H}_{\mathrm{d}}$ proton at $\delta 1.90$ results in partial collapse of the multiplet at $\delta 3.05\left(\mathrm{H}_{\mathrm{b}}\right.$ and $\mathrm{H}_{\mathrm{c}}$ protons). The above data strongly confirm the regiochemistry of $\mathbf{4 a , b}$.
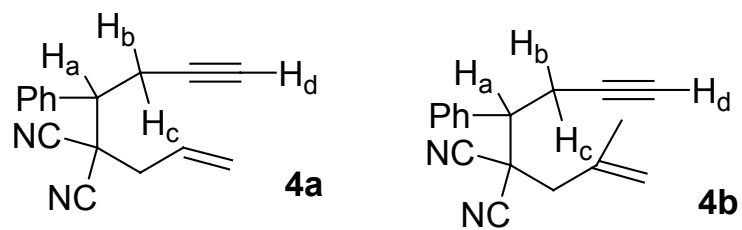

Similarly, irradiation of the $\mathrm{H}_{\mathrm{a}}$ proton at $\delta 3.43$ in $\mathbf{6 b}$ led to partial collapse of the multiplet at $\delta 2.89\left(\mathrm{H}_{\mathrm{b}}\right.$ and $\mathrm{H}_{\mathrm{c}}$ protons). Irradiation of the two protons $\left(\mathrm{H}_{\mathrm{b}}\right.$ and $\left.\mathrm{H}_{\mathrm{c}}\right)$ multiplet at $\delta 2.89$ converts the doublet of a doublet at $\delta 3.43$ to a sharp singlet and also the triplet at $\delta 1.71\left(\mathrm{H}_{\mathrm{d}}\right.$ proton) to a sharp singlet. Irradiation of the $\mathrm{H}_{\mathrm{d}}$ proton at $\delta 1.71$ results in partial collapse of the multiplet at $\delta 3.43\left(\mathrm{H}_{\mathrm{b}}\right.$ and $\mathrm{H}_{\mathrm{c}}$ protons).

Similarly, irradiating the $\mathrm{H}_{\mathrm{a}}$ proton at $\delta 3.73$ in 8a, the multiplet at $\delta 3.05\left(\mathrm{H}_{\mathrm{b}}\right.$ and $\mathrm{H}_{\mathrm{c}}$ protons) collapses partially. Irradiation of the two protons $\left(\mathrm{H}_{\mathrm{b}}\right.$ and $\left.\mathrm{H}_{\mathrm{c}}\right)$ multiplet at $\delta 3.05$ causes the signal at $\delta 3.73$ (doublet of a doublet) to a sharp singlet and also converts the triplet at $\delta 1.74\left(\mathrm{H}_{\mathrm{d}}\right.$ proton) to a sharp singlet. Irradiation of the $\mathrm{H}_{\mathrm{d}}$ proton at $\delta 1.74$ leads to partial collapse of the multiplet at $\delta 3.05\left(\mathrm{H}_{\mathrm{b}}\right.$ and $\mathrm{H}_{\mathrm{c}}$ protons). The above data strongly confirm the regiochemistry of $\mathbf{6 b}$ and $\mathbf{8 a}$. 


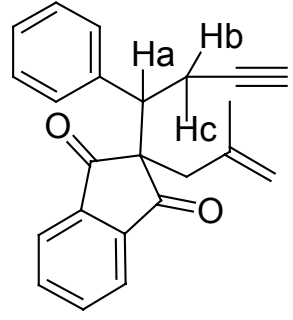

$6 b$

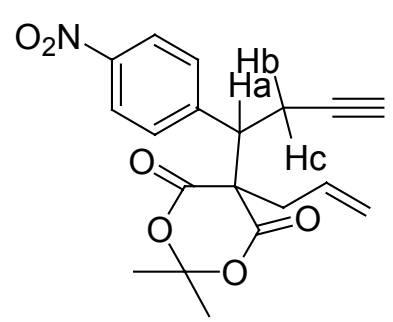

$8 a$

The structures of 1,2-addition products $\mathbf{1 0 a}, \mathbf{b}, \mathbf{e}, \mathbf{g}$ were established by ${ }^{1} \mathrm{H}$ NMR NOE experiments. For example, selective irradiation of the $H_{a}$ proton at $\delta 6.67$ in 10a caused $2.32 \%$ enhancement of the ortho protons of the phenyl ring at $\delta 7.30$. Selective irradiation of the $\mathrm{H}_{\mathrm{b}}$ proton at $\delta 6.00$ caused $3.30 \%$ enhancement of the ortho protons at $\delta$ 7.30. Similar results were obtained for $\mathbf{1 0 b}, \mathbf{e}, \mathbf{g}$ and were shown below. These NOE results strongly confirm the structures of $\mathbf{1 0 a}, \mathbf{b}, \mathbf{e}, \mathbf{g}$.
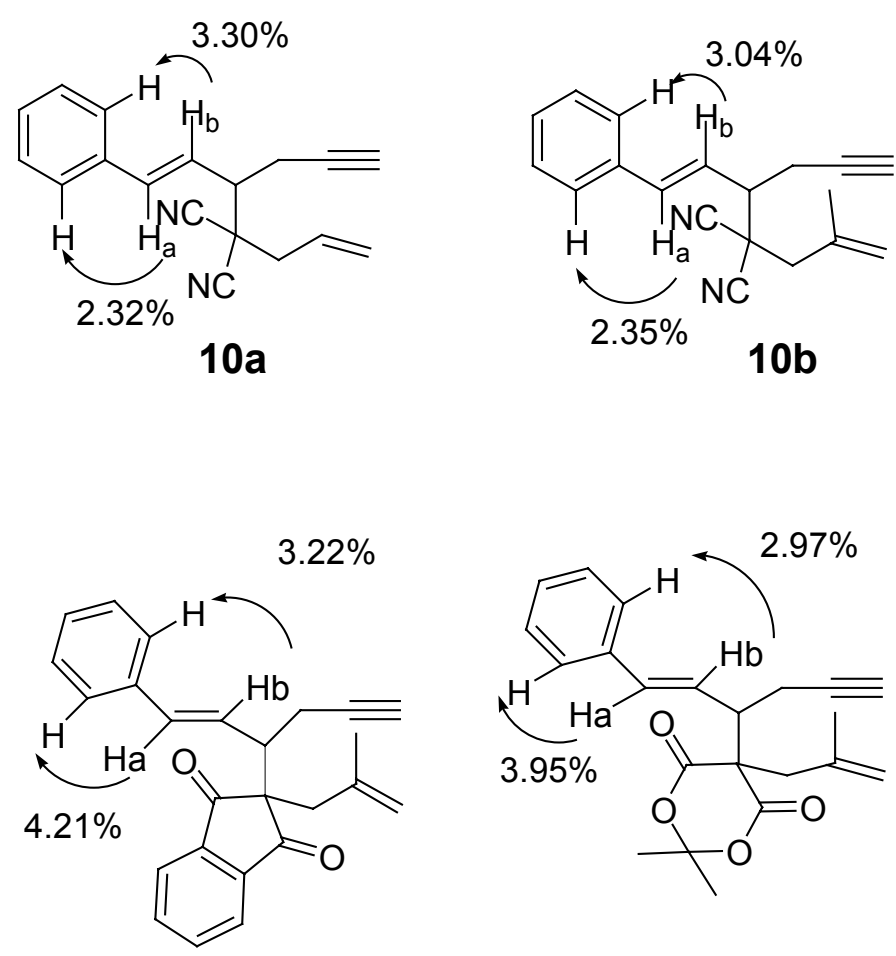

$10 \mathrm{e}$

$10 \mathrm{~g}$ 


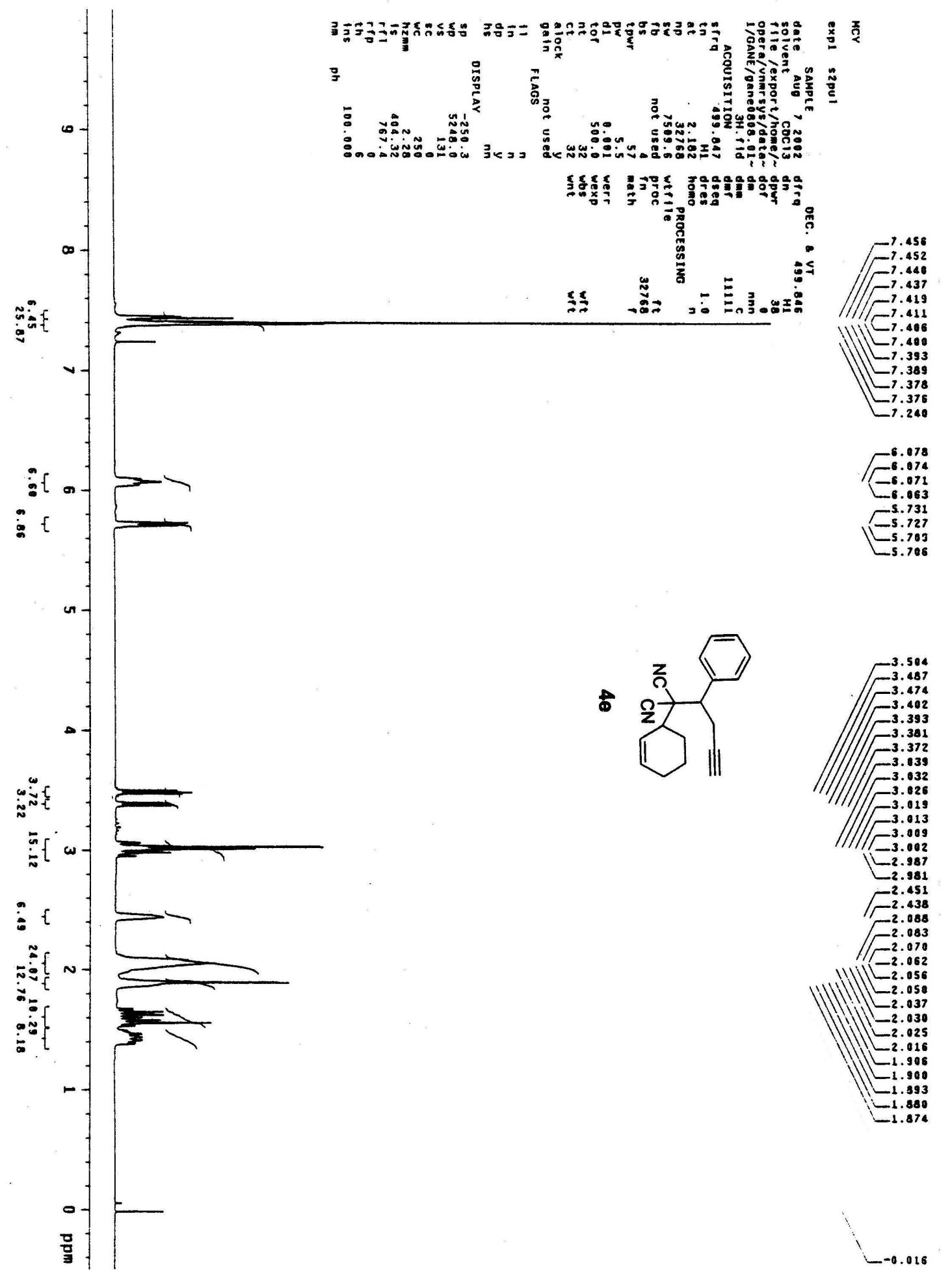




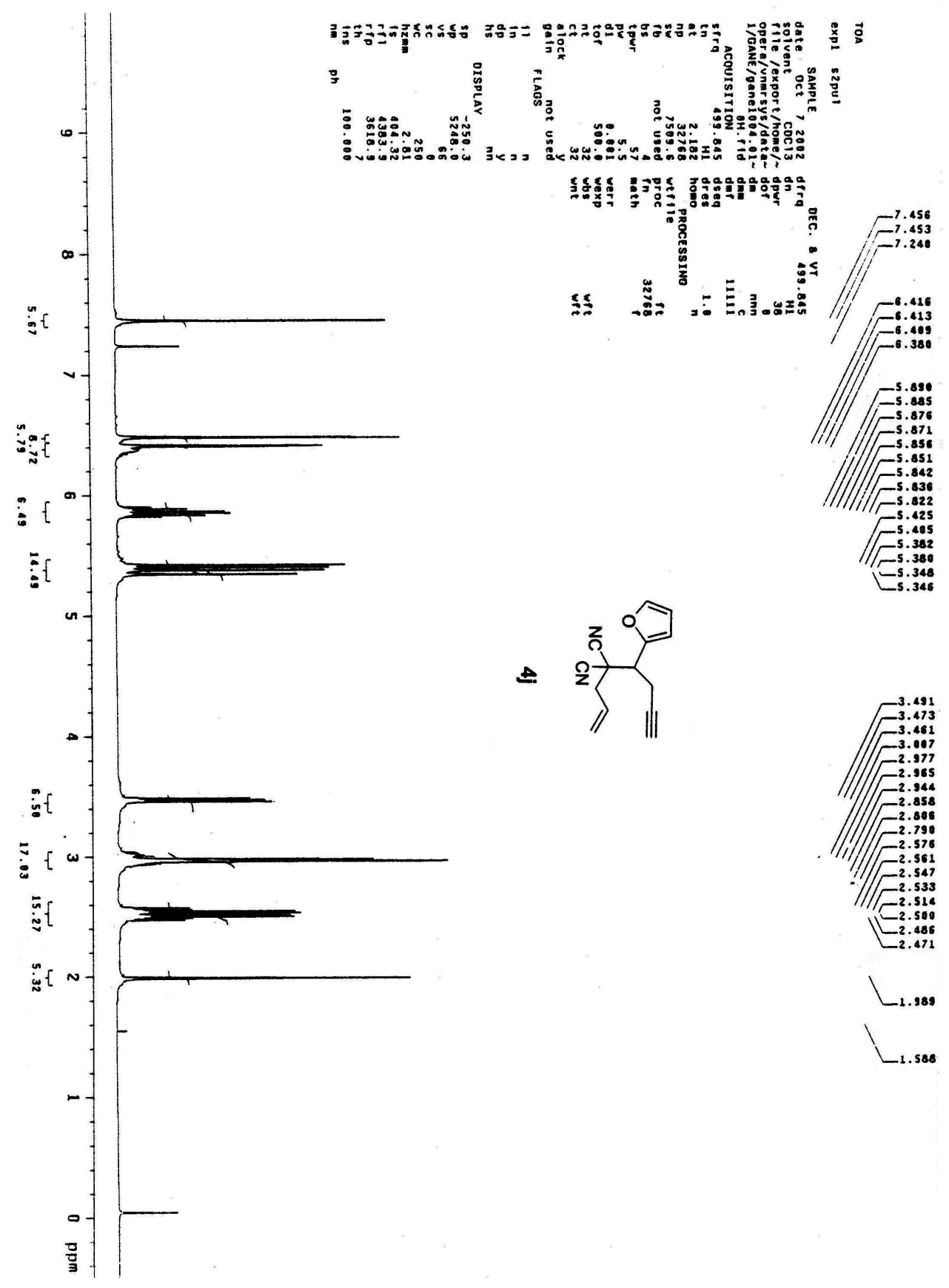




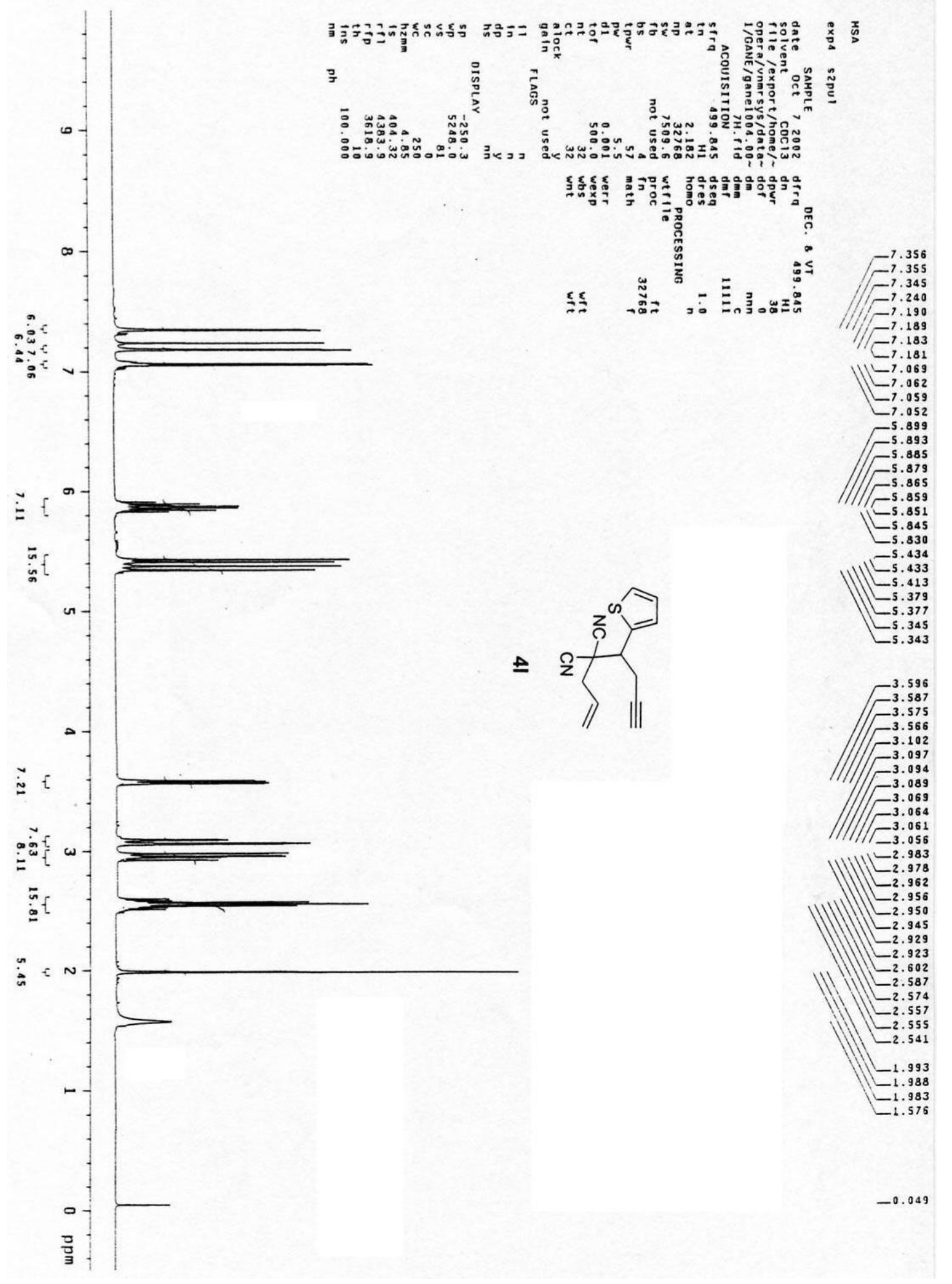



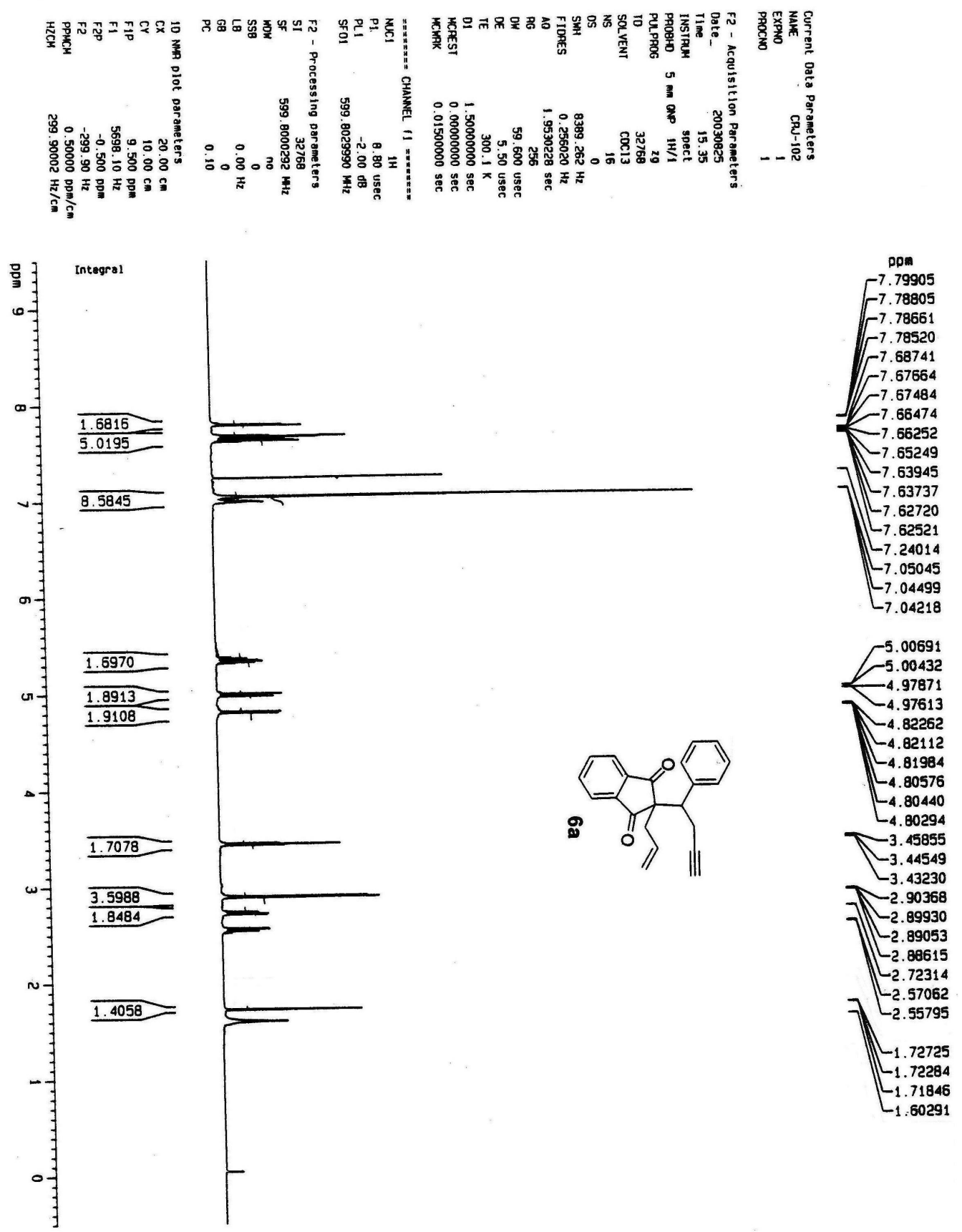


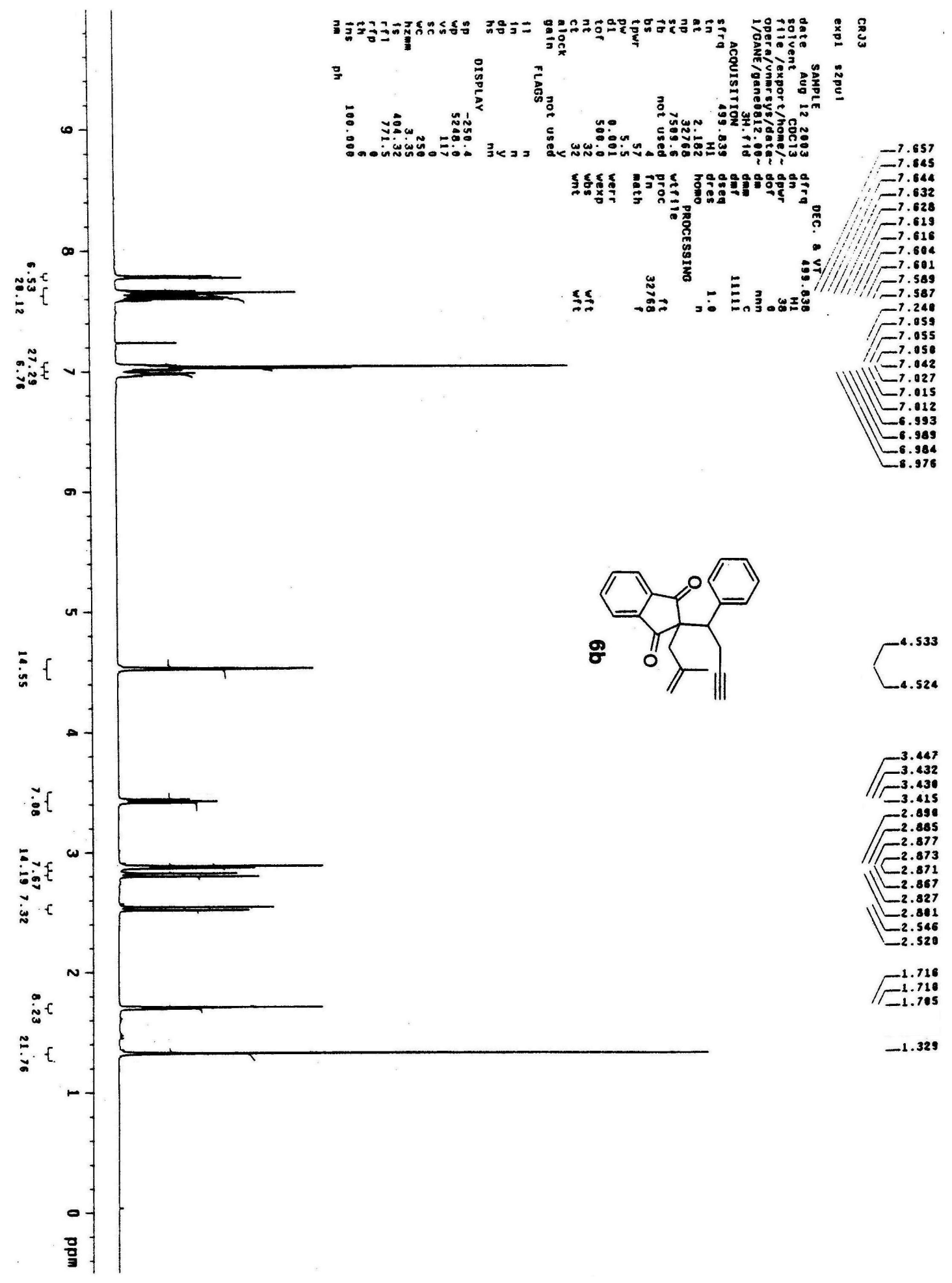



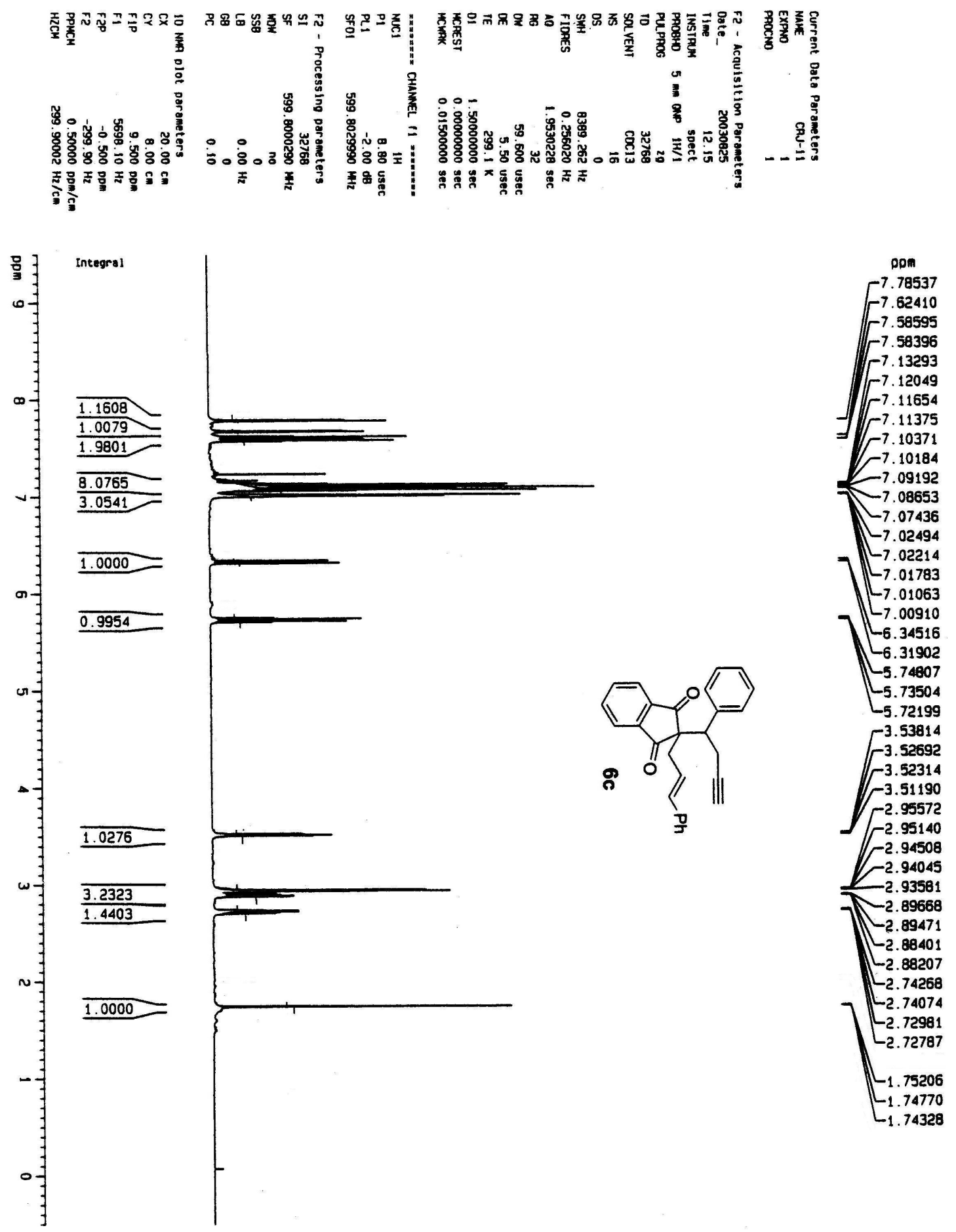

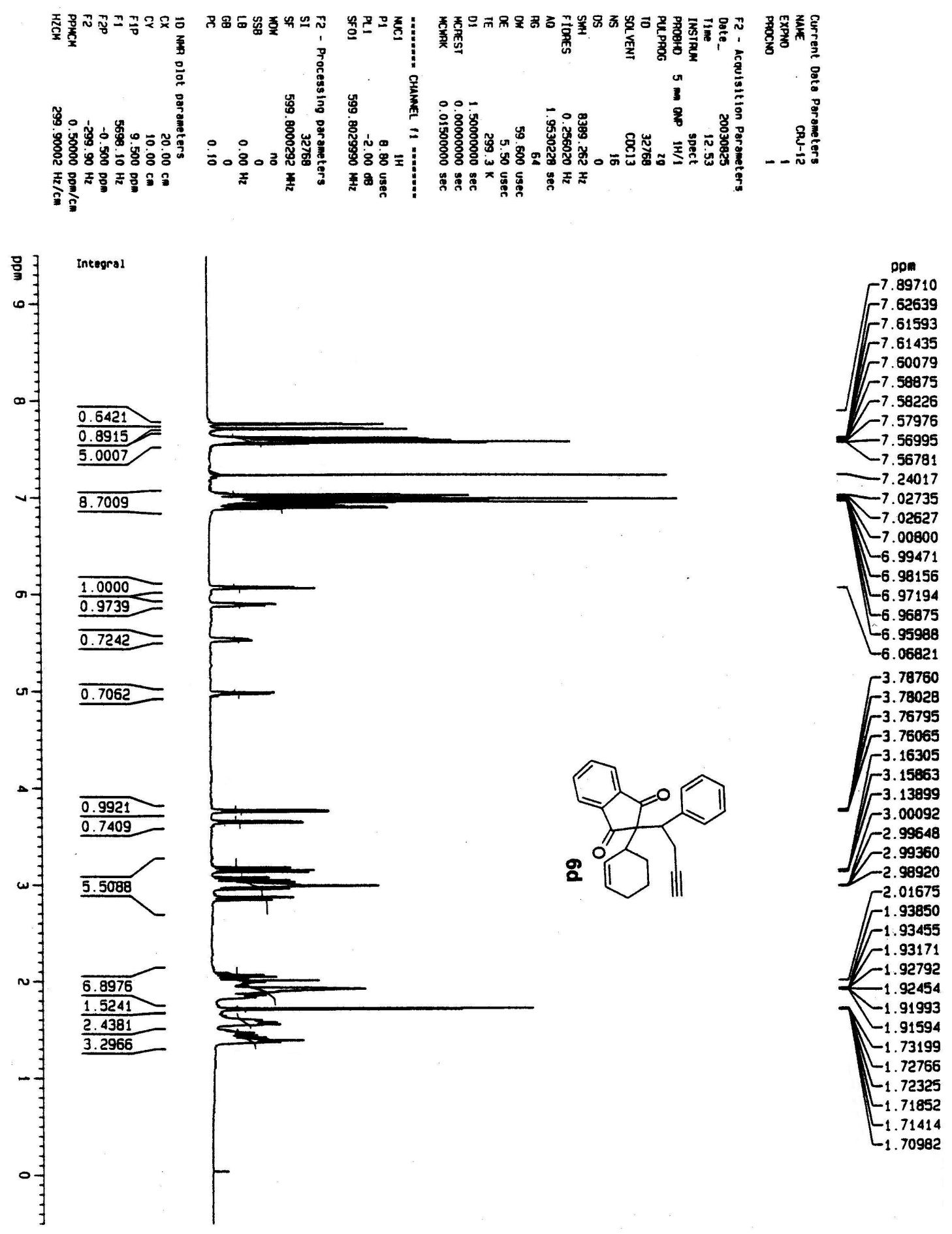


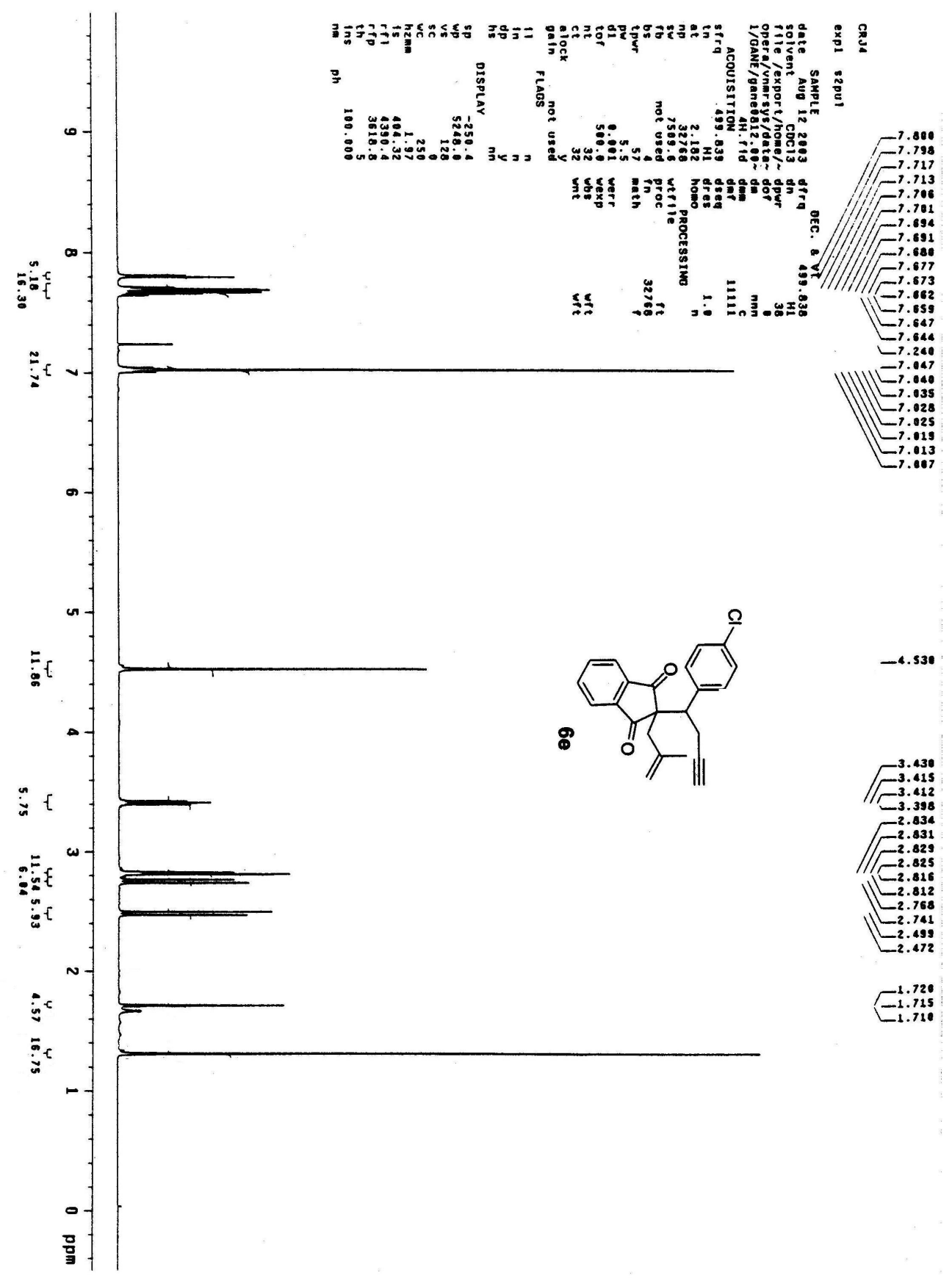



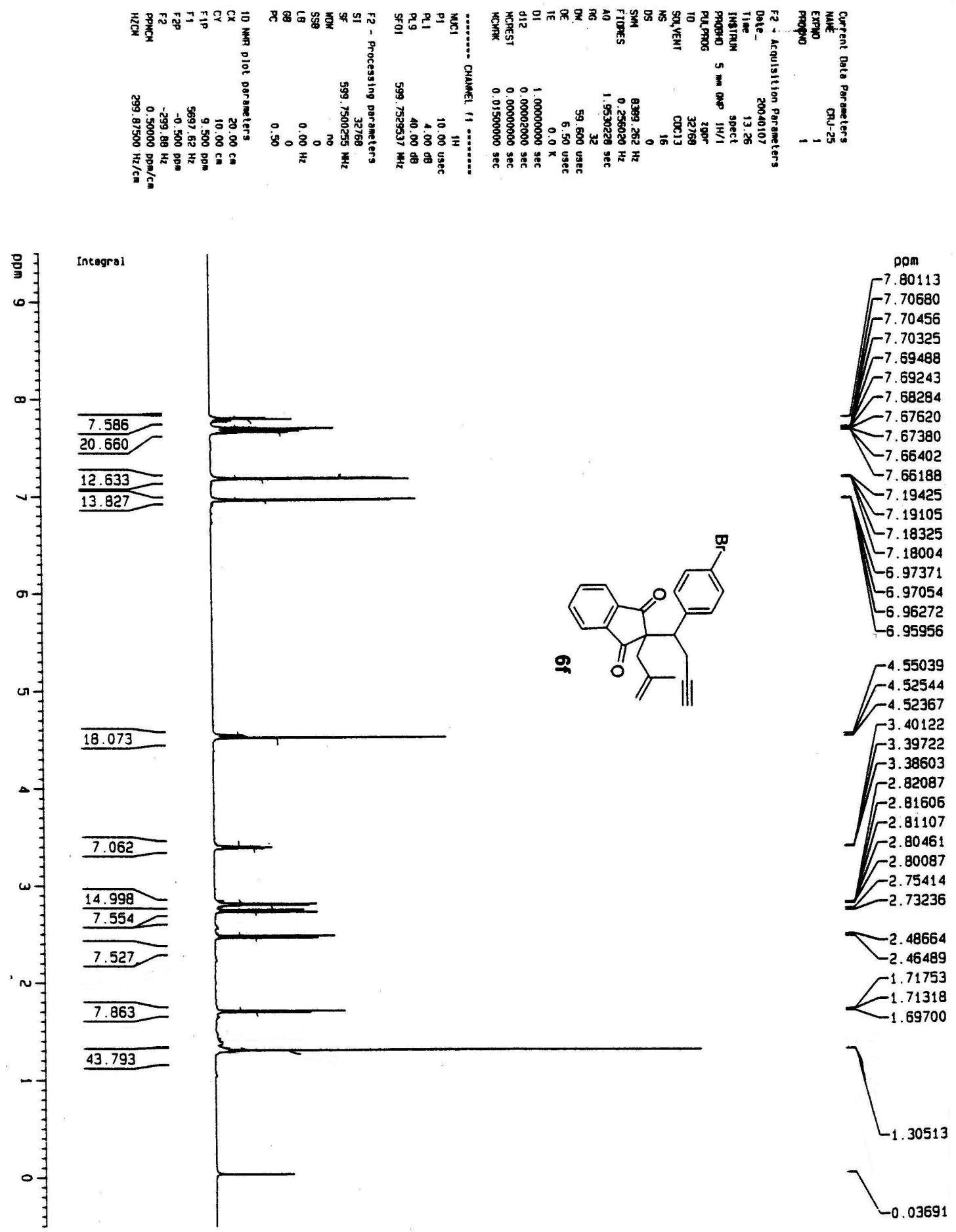


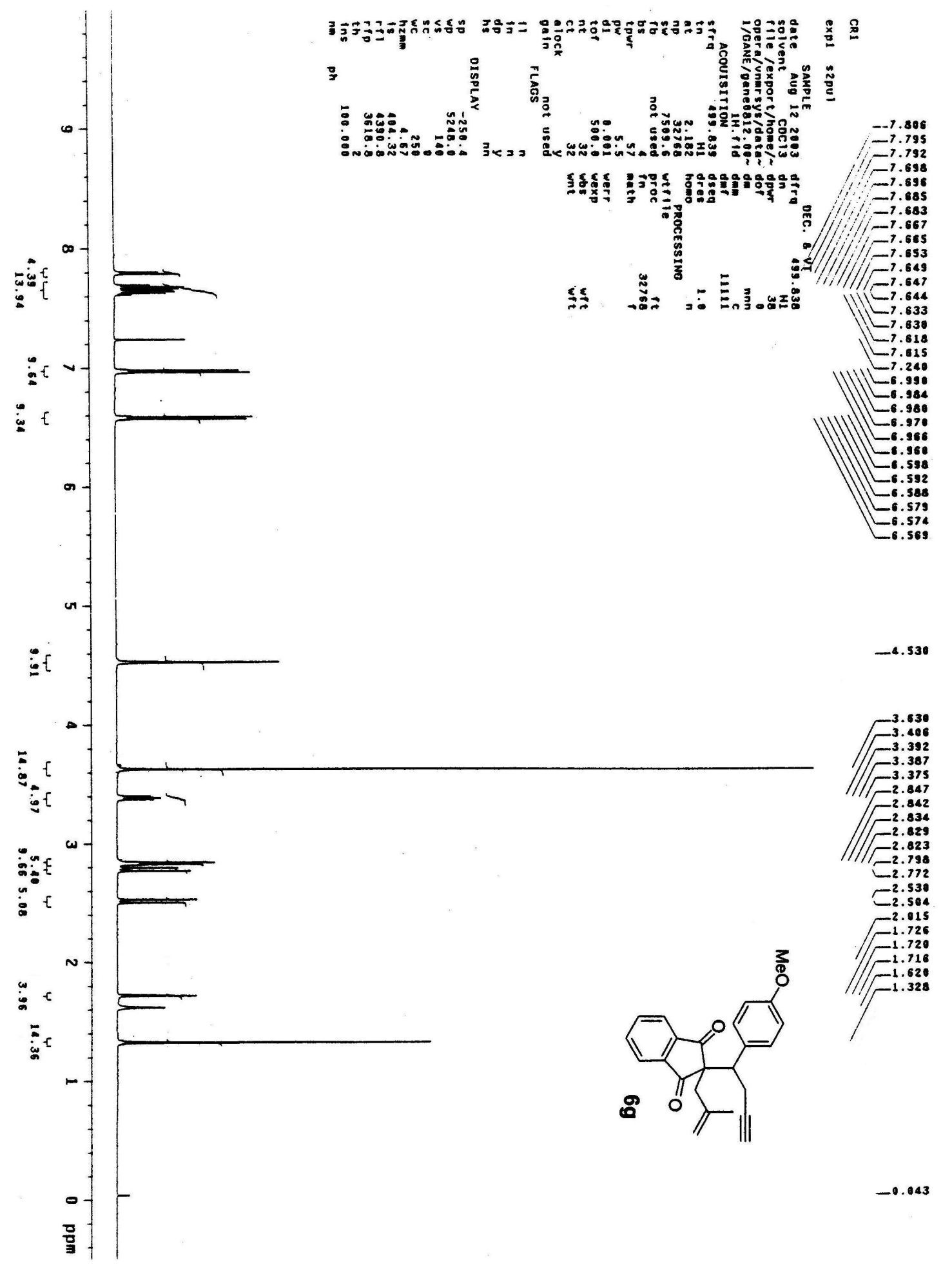




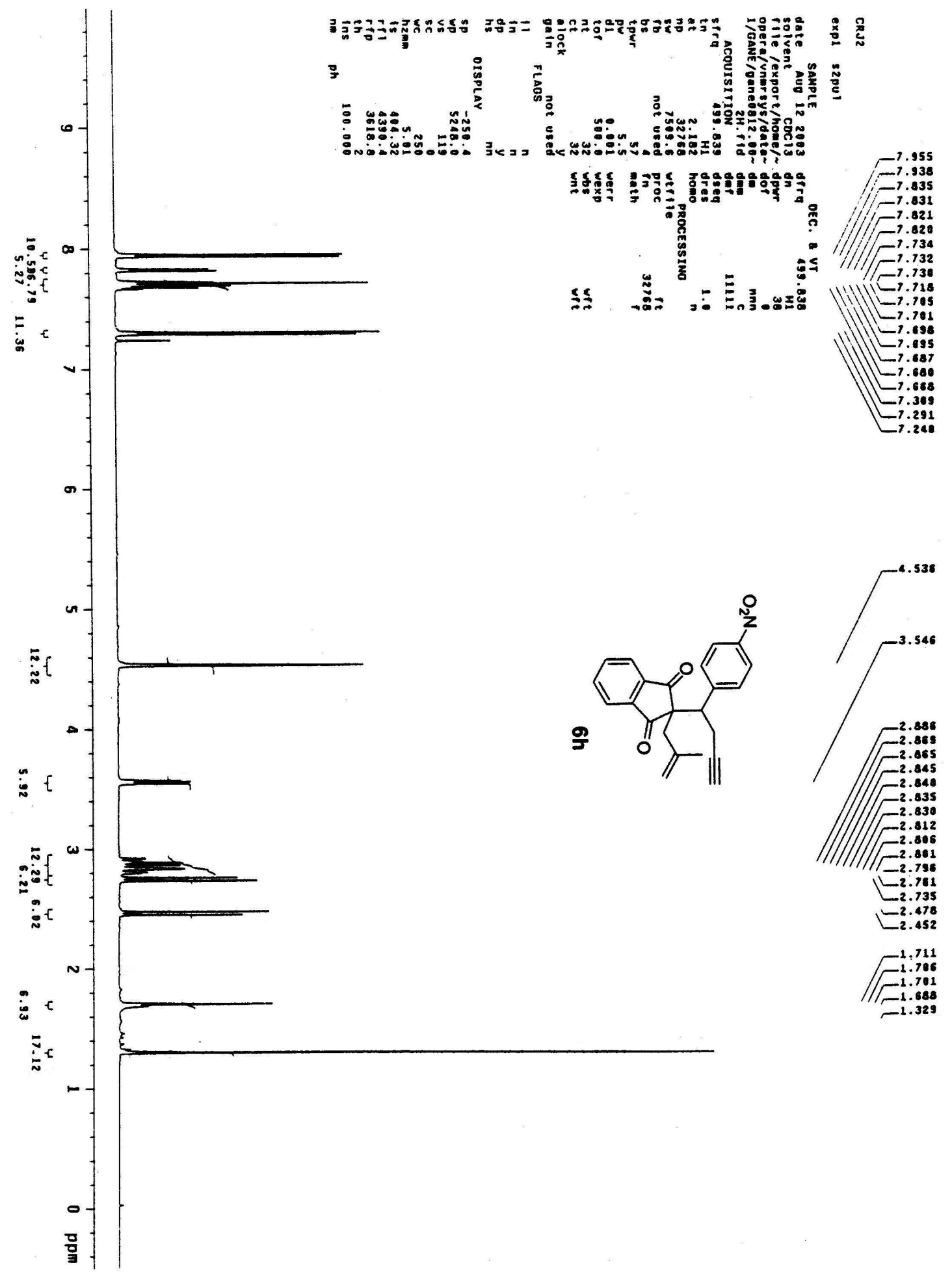



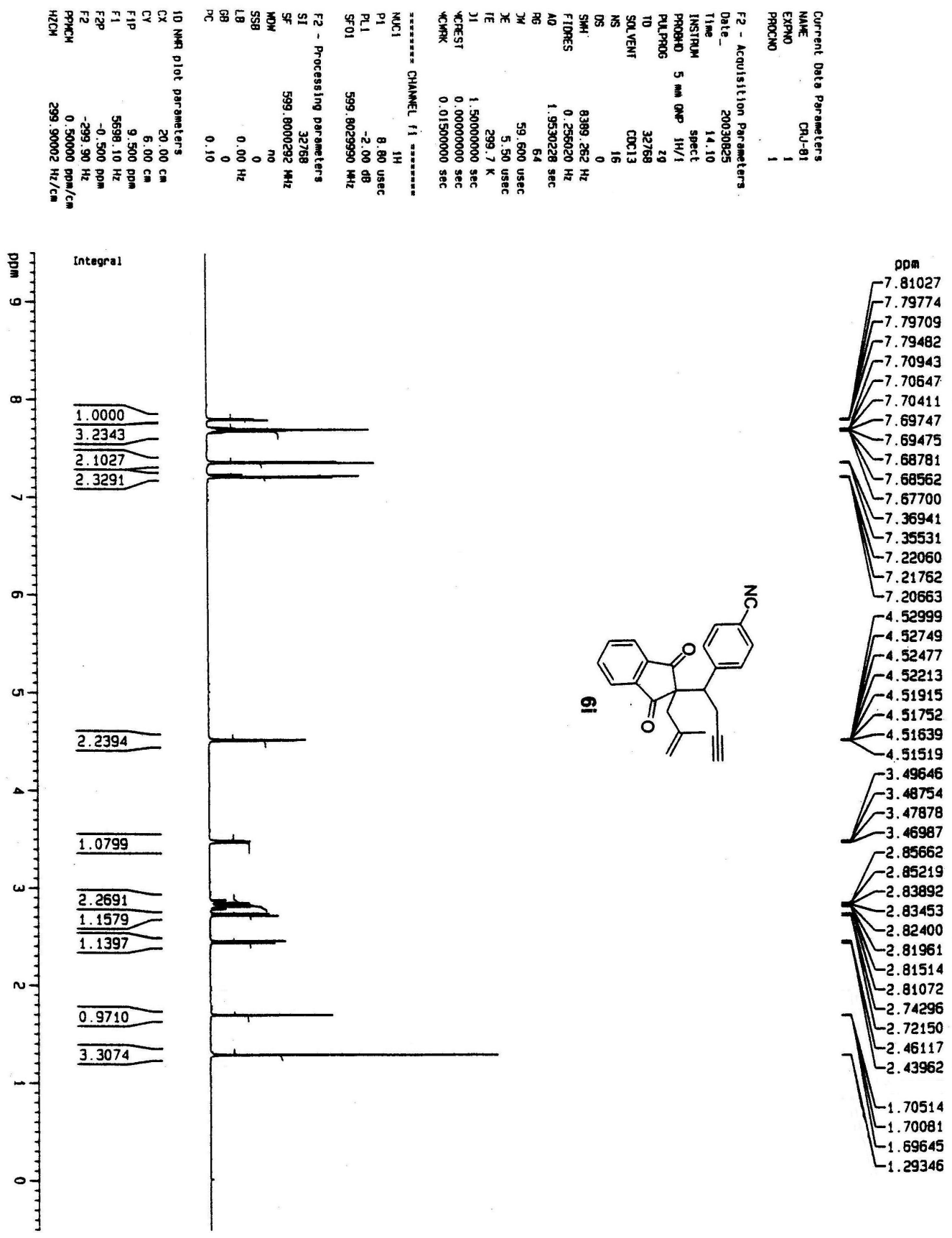


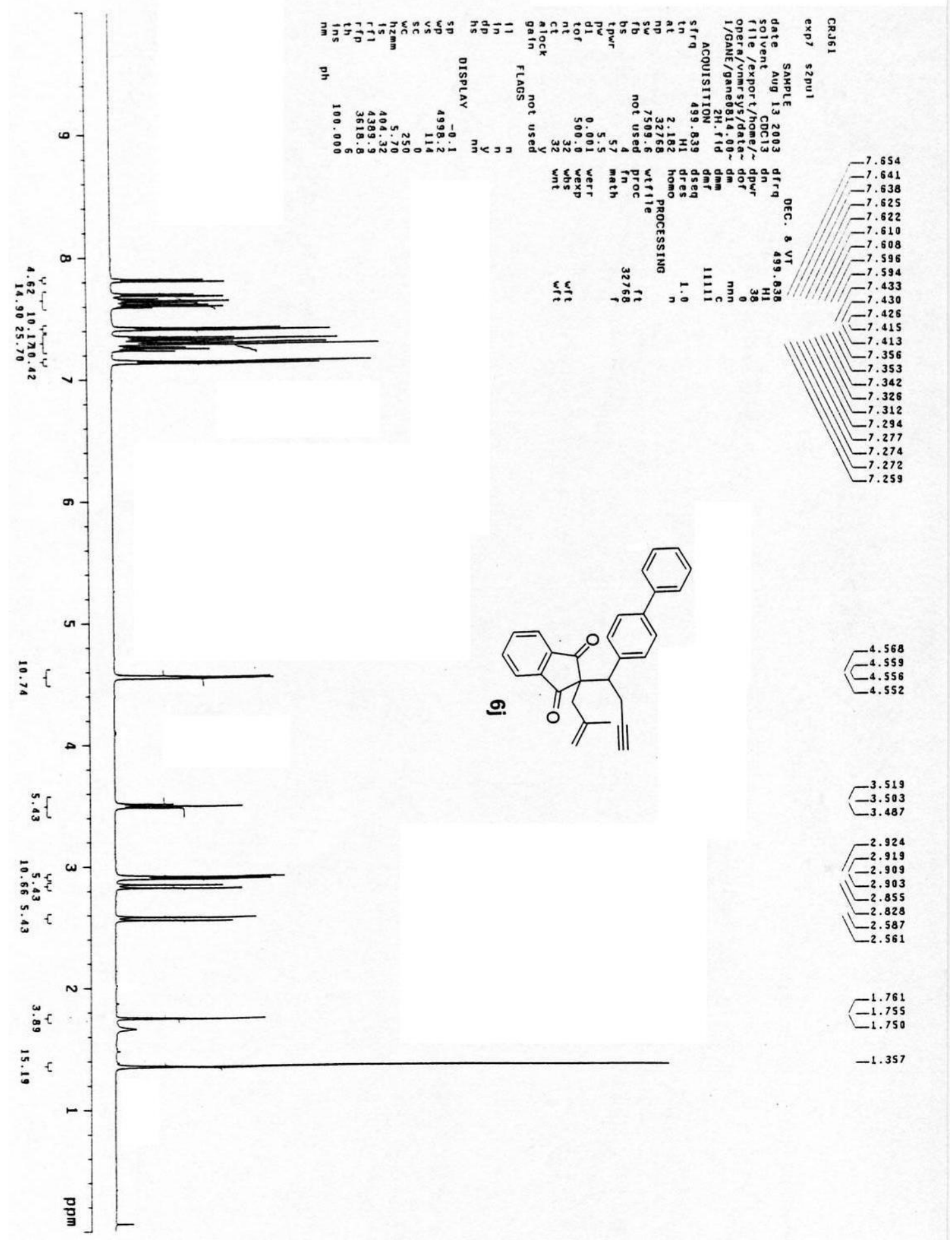




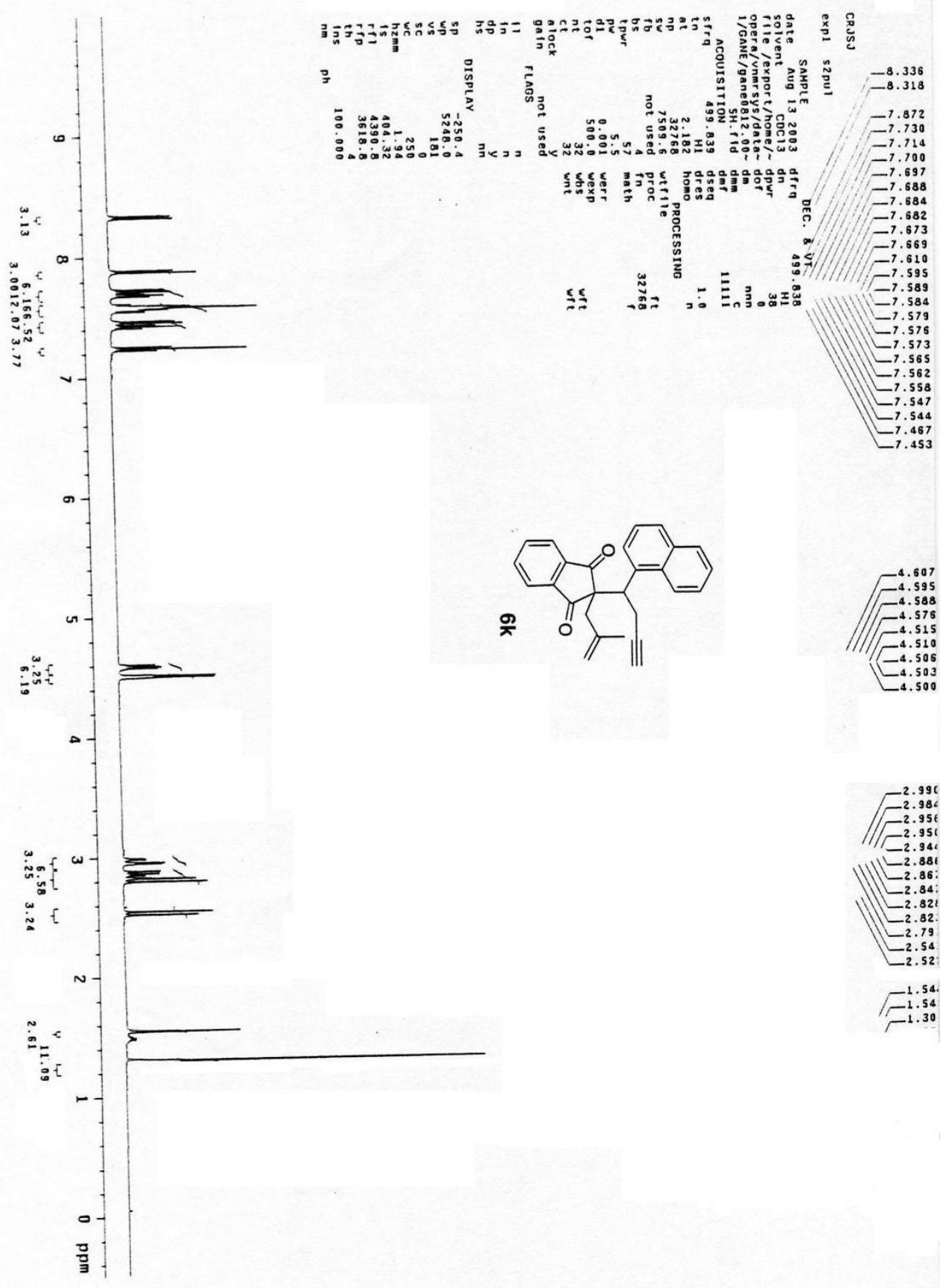



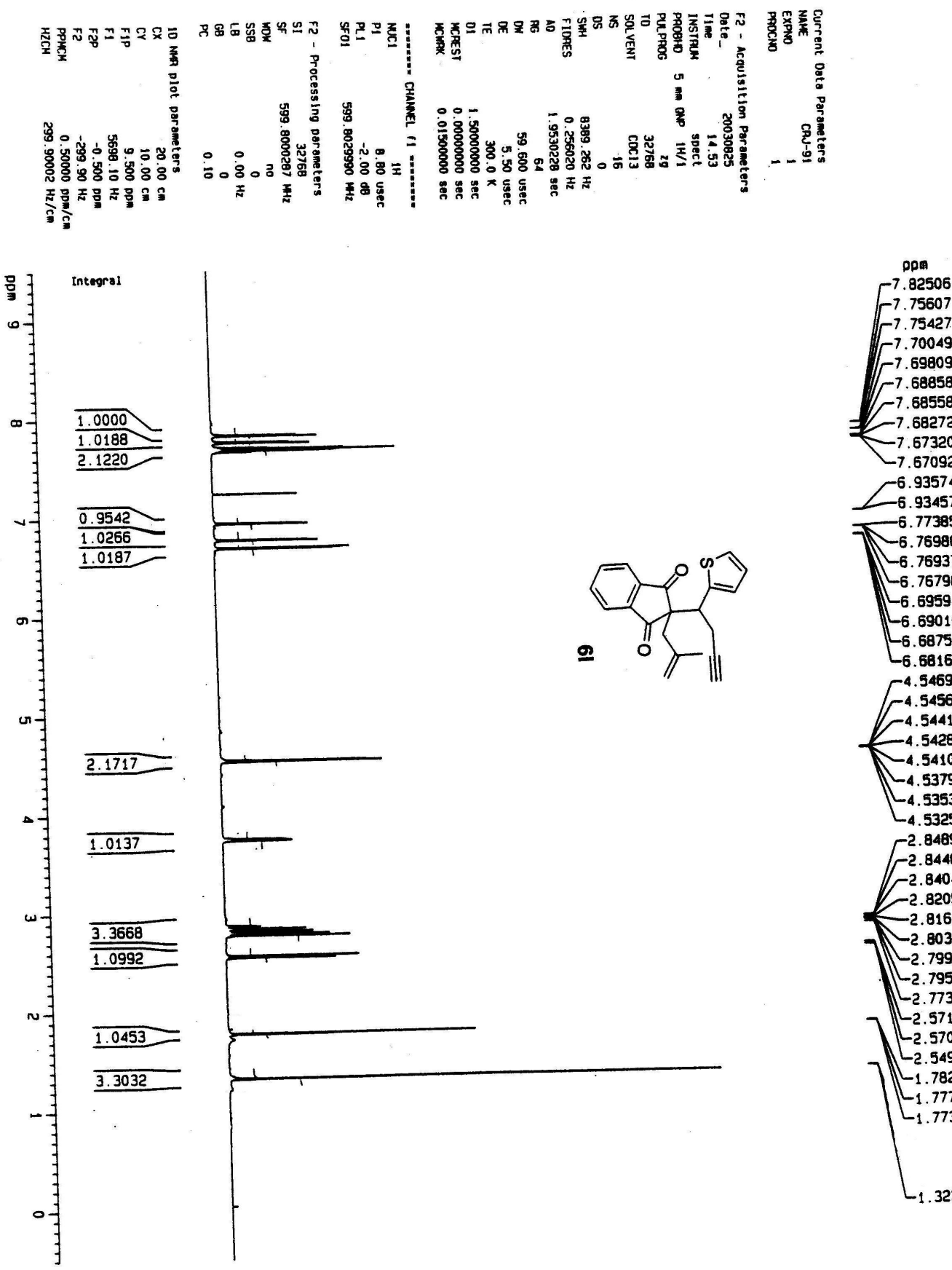

-6. 65989

$-6.76937$

$-6.76798$

$-6.6959$

-6.69010

$L_{6} .68750$

$-6.68163$

$\Gamma^{4.54695}$

$-4.54412$

$-4.54106$

$-4.53799$

$-4.53533$

$-4.53250$

2. 84899

2.84048

$-2.82051$

$-2.81613$

$-2.80342$

$-2.79902$

$-2.79504$

$-2.77388$

$-2.5710$

$-2.57053$

$-2.54926$

$L_{-1.78203}$

$L_{1.77768}$

$L_{1.77330}$

$L_{1.32371}$ 


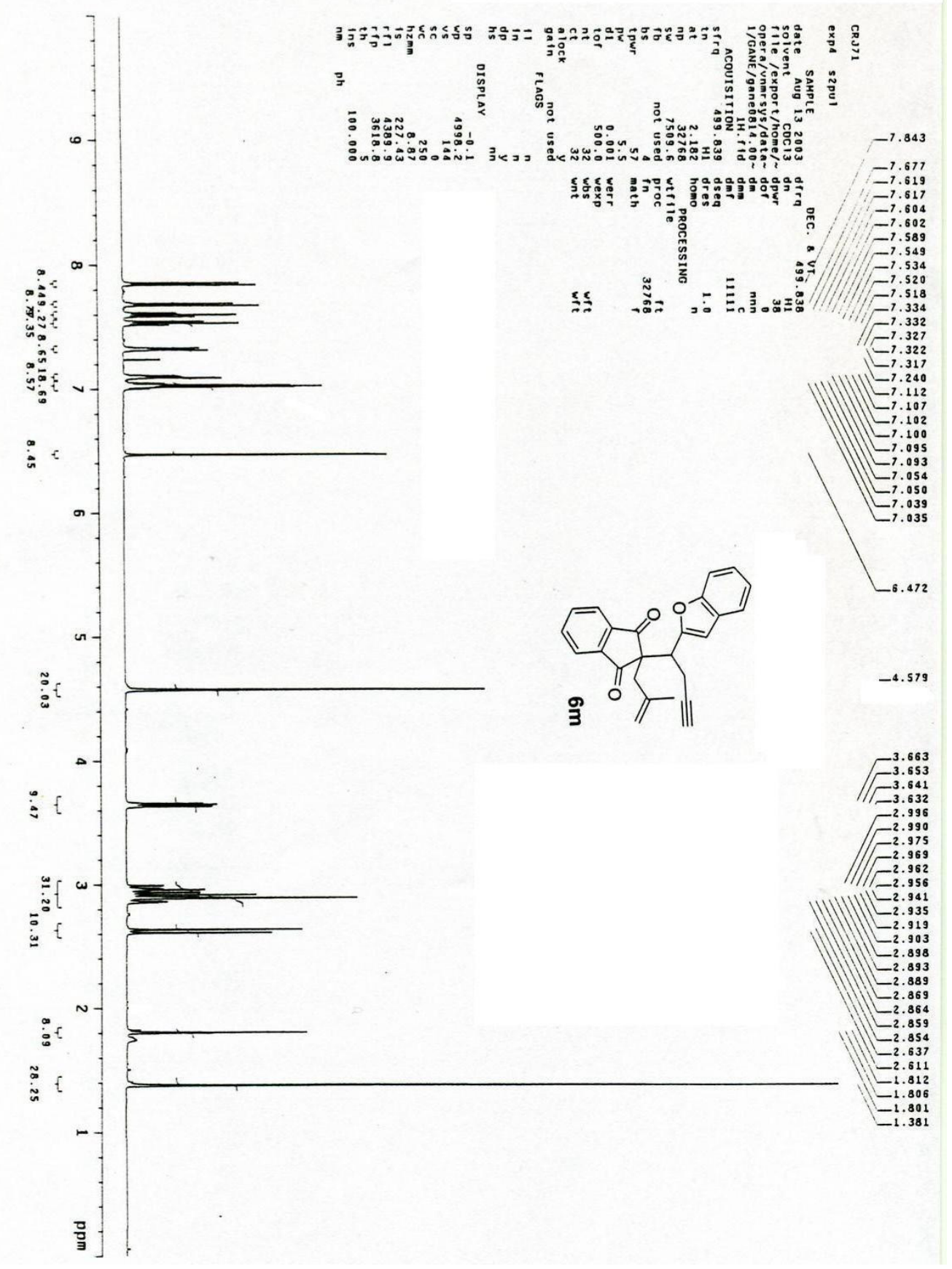



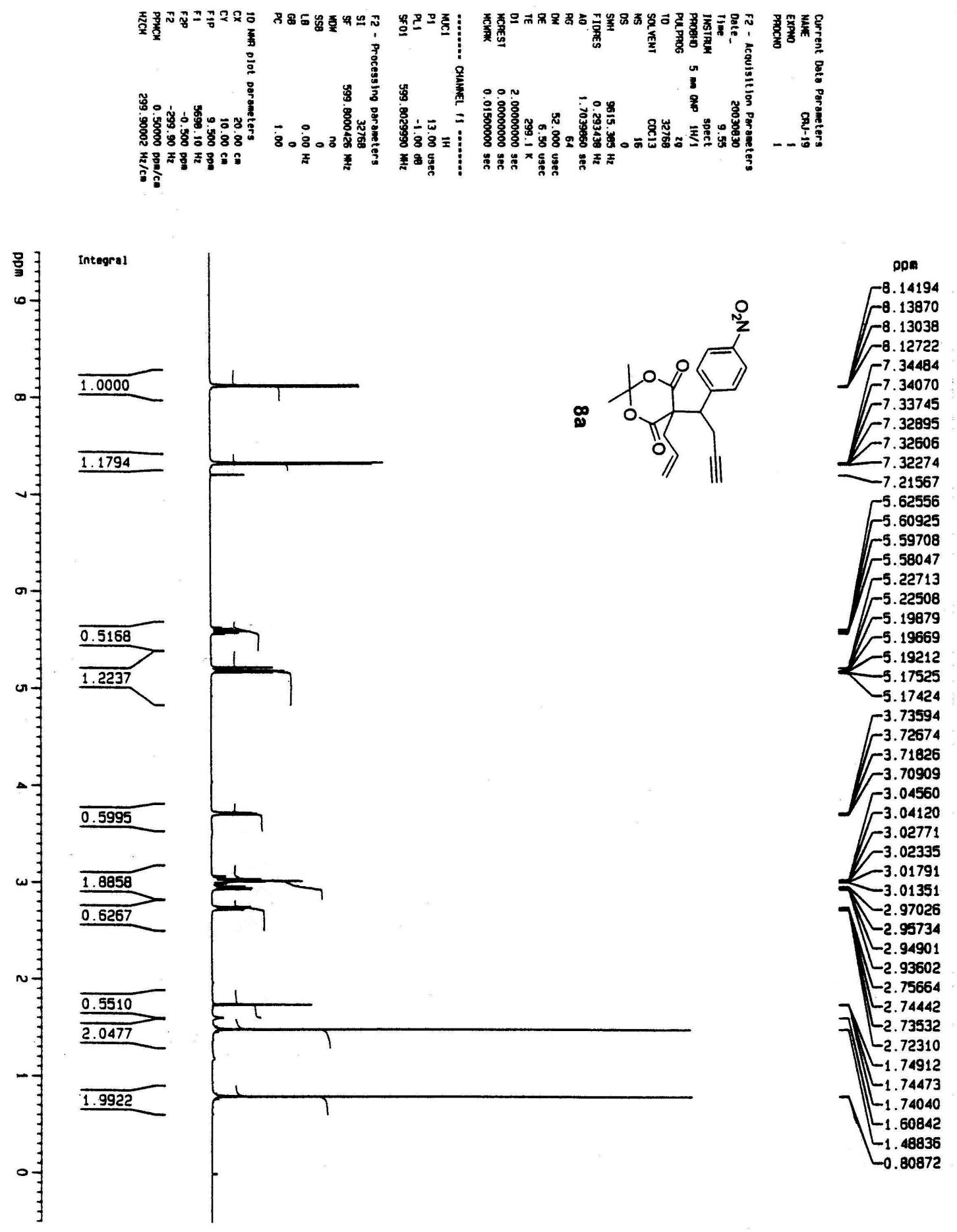

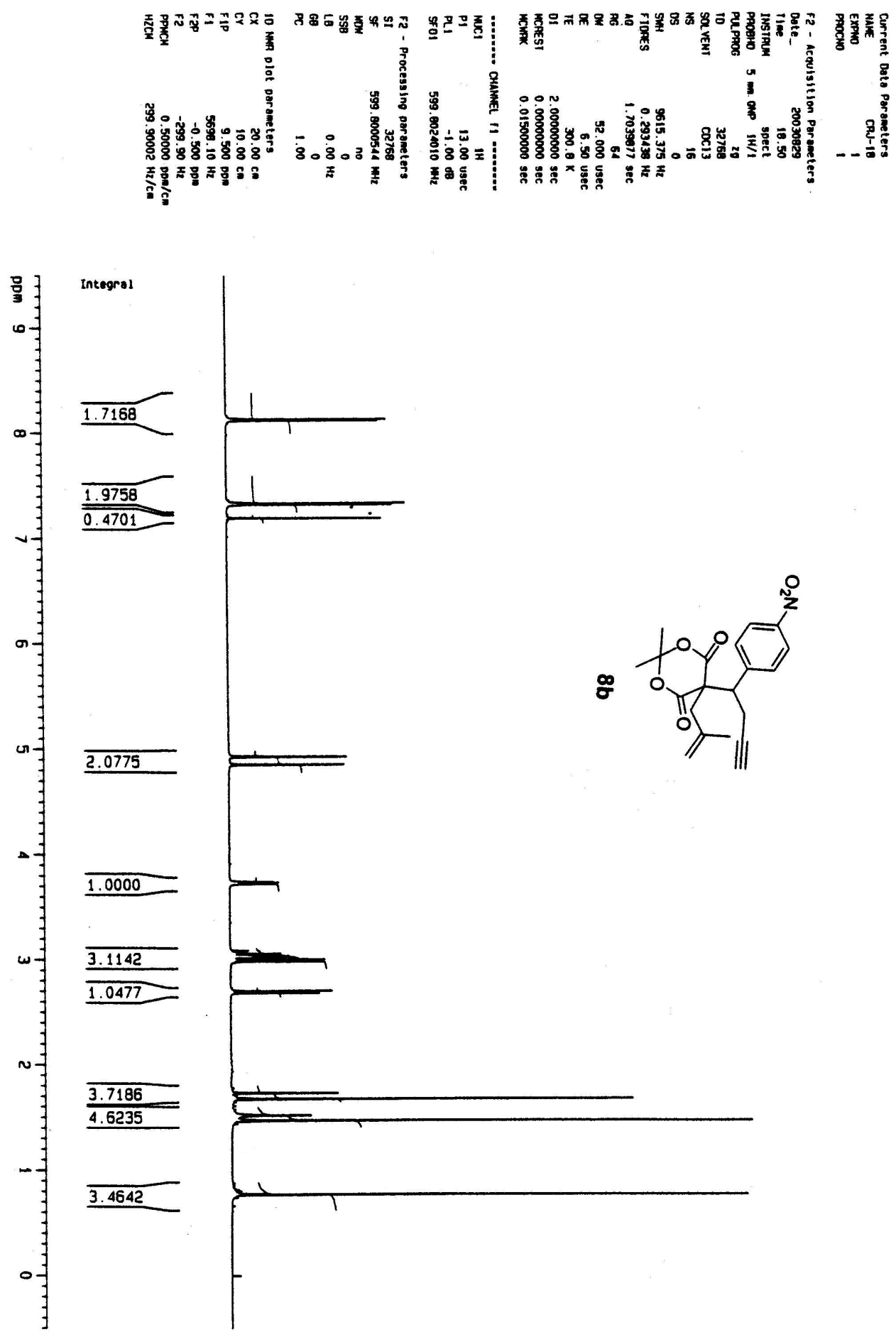

Dอต

8.14273

8.13871

${ }^{8.13546}$

8.13218

$-8.11983$

7.34304

7.33564

$-7.32421$

-7.19697

-7.19571

4.93248

$r^{4.93006}$

$r^{4.85612}$

$-3.74843$

-3.73966

$-3.73041$

$r^{3.72168}$

$-3.09147$

$-3.08709$

$-3.06899$

$-3.06295$

$-3.05856$

$-3.04490$

3.04044
-3.03720

$-3.03722$

$-3.03280$

-02843
-3.02404

$-3.00501$

$-2.99993$

$-2.98434$

$-2.70504$

$-2.68425$

$-1.73678$

$-1.73239$

$L_{1.72804}$

$-1.67498$

$-1.51994$

$-1.46998$

$L_{-1.18570}$

$L_{0.76893}$ 

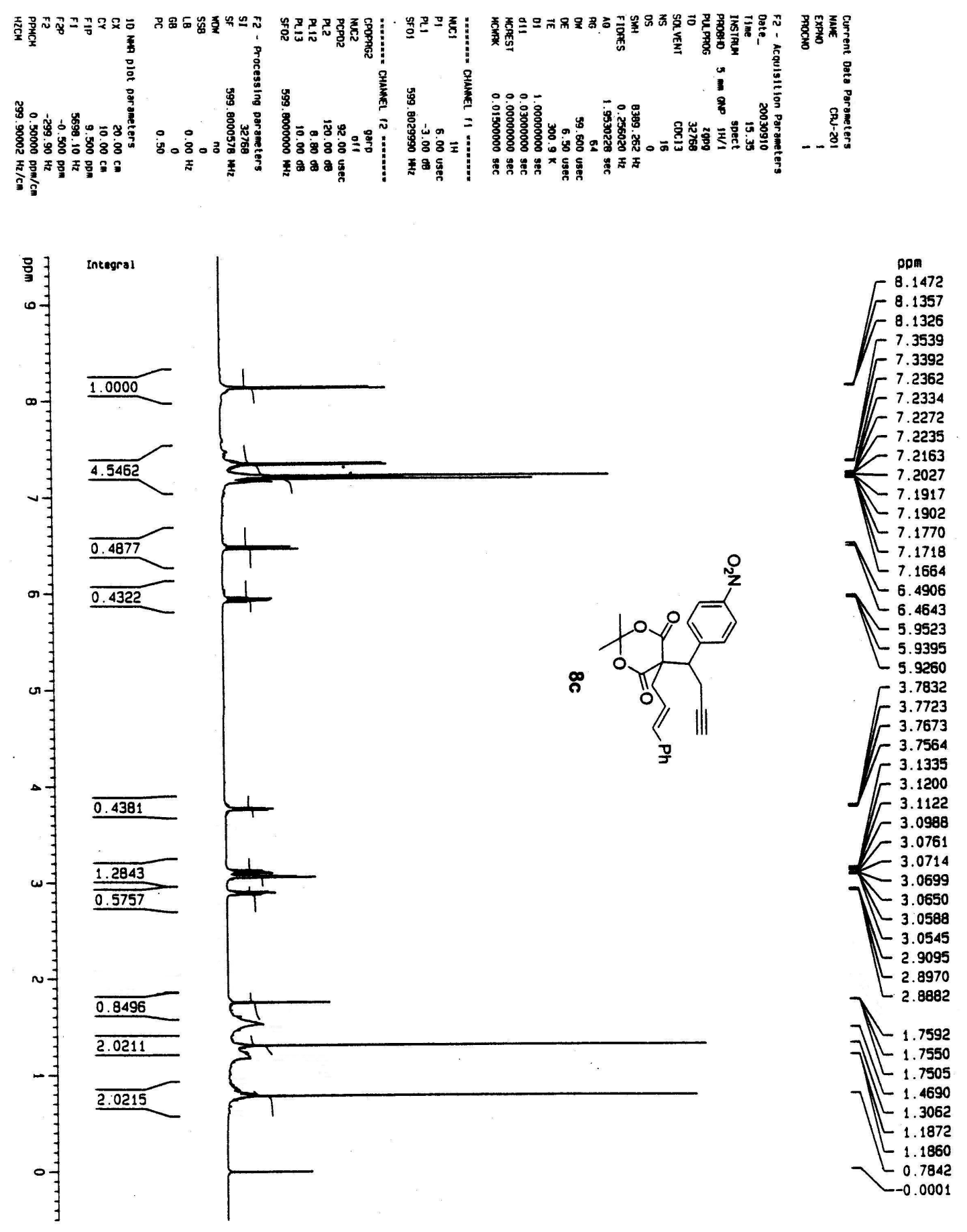

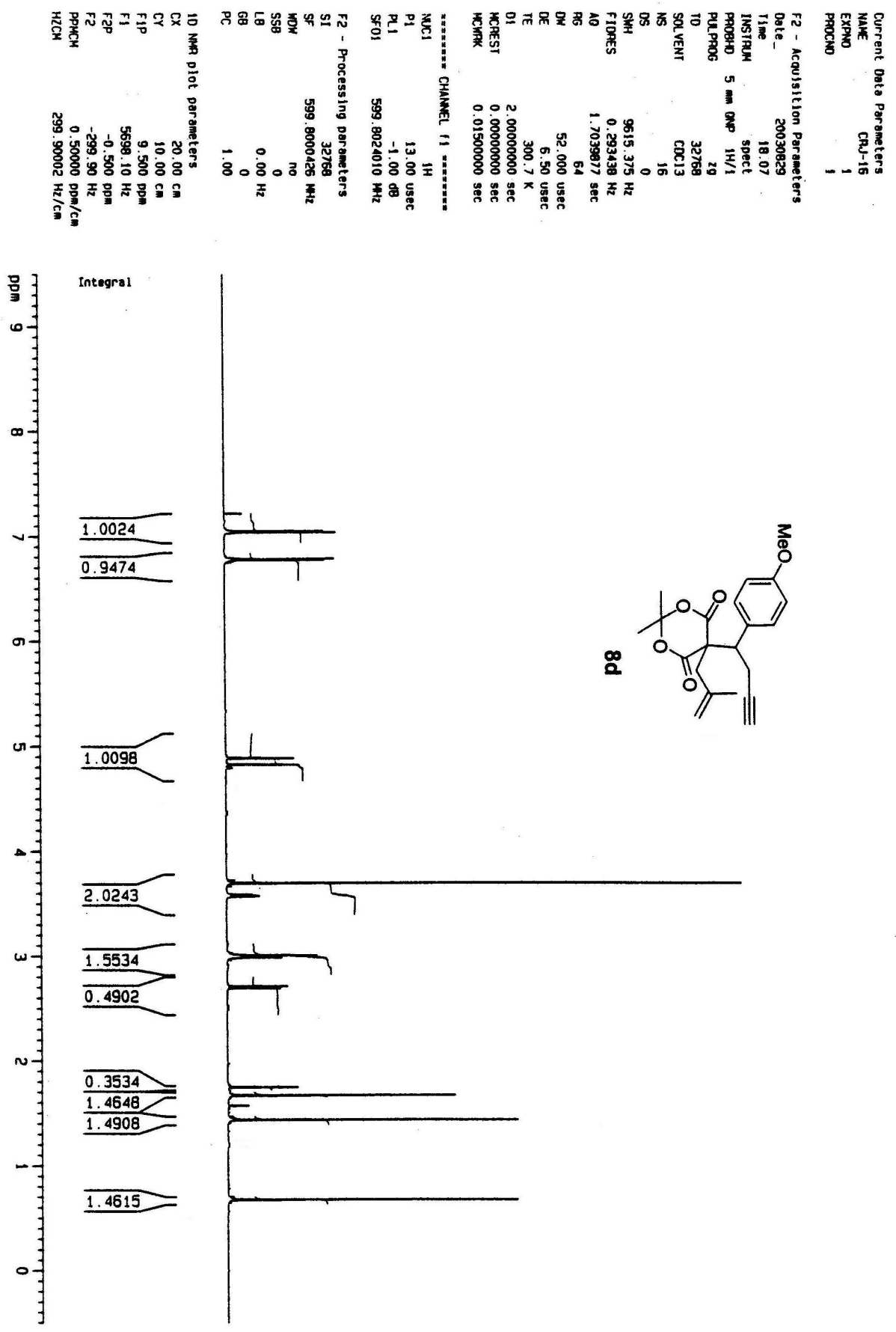

pDm

7.21655

$\overbrace{}^{7.04765}$

$\begin{array}{r}7.03632 \\ -7.03306 \\ \hline\end{array}$

$-6.78225$

$-6.7708$

-6.77088
-6.76762

4.88750

$-4.88490$

$-4.82239$

3.70050

-3.69401

3.58994

-3.57936

$-3.57419$

3.56359

$-3.00720$

$-3.00239$

$-2.99804$

$-2.99346$

$-2.98662$

$-2.98071$

$-2.70799$

$-2.68717$

$-1.74643$

$-1.74208$

$\left[\begin{array}{l}-1.73773 \\ -1.70542\end{array}\right.$

$L_{1} .65354$

$-1.56658$

$L_{1.43201}$

$L_{1.20406}$

$-0.67077$ 

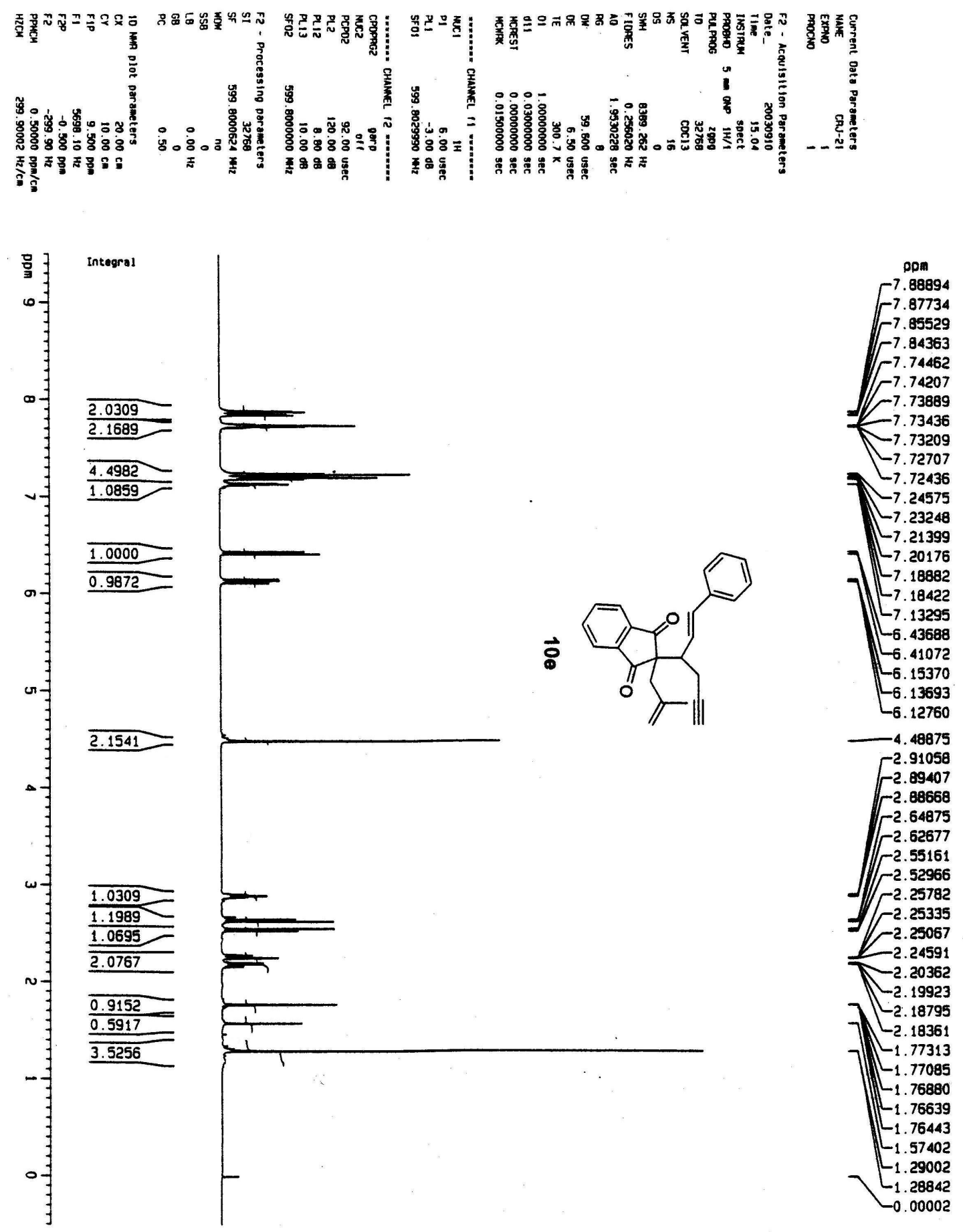

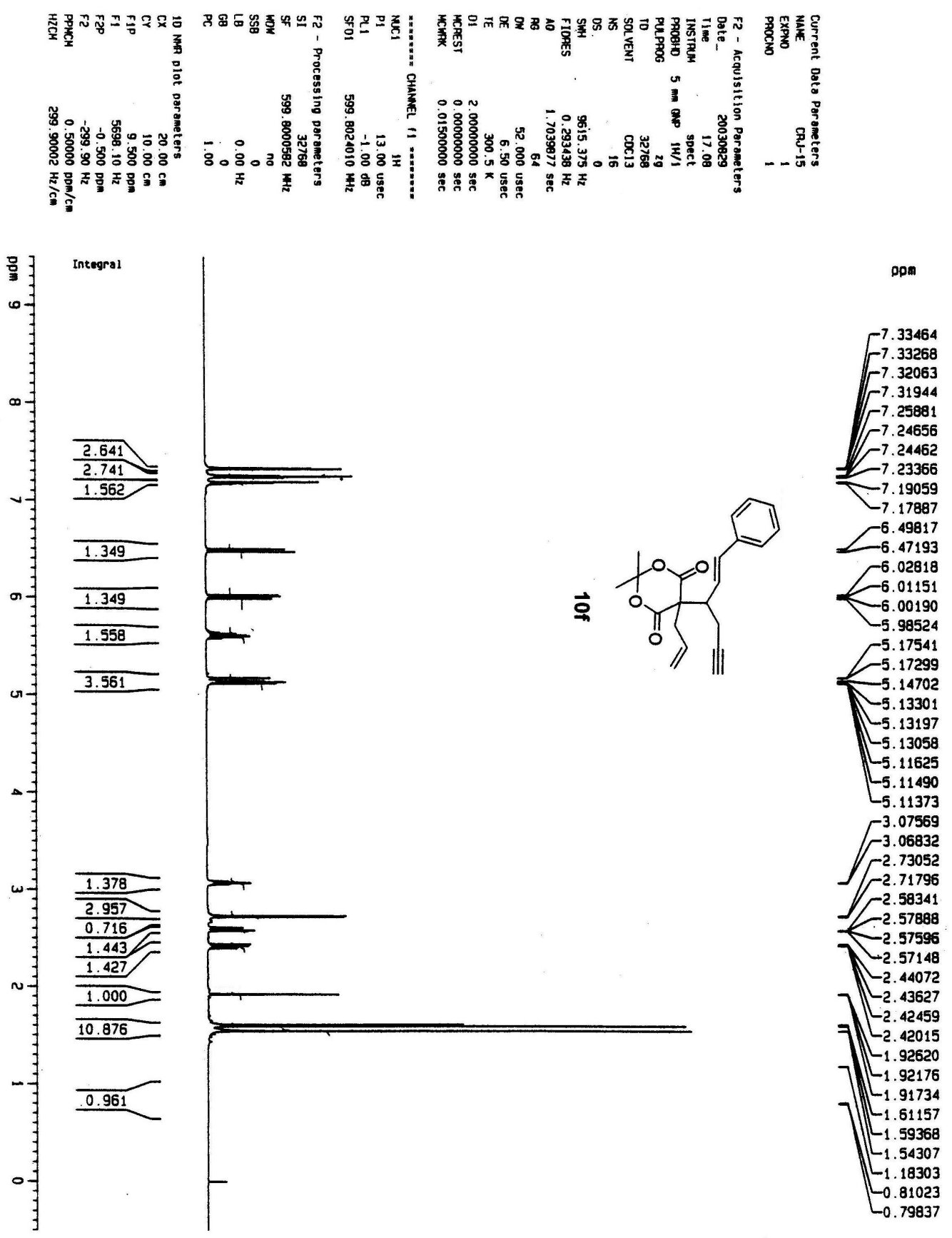

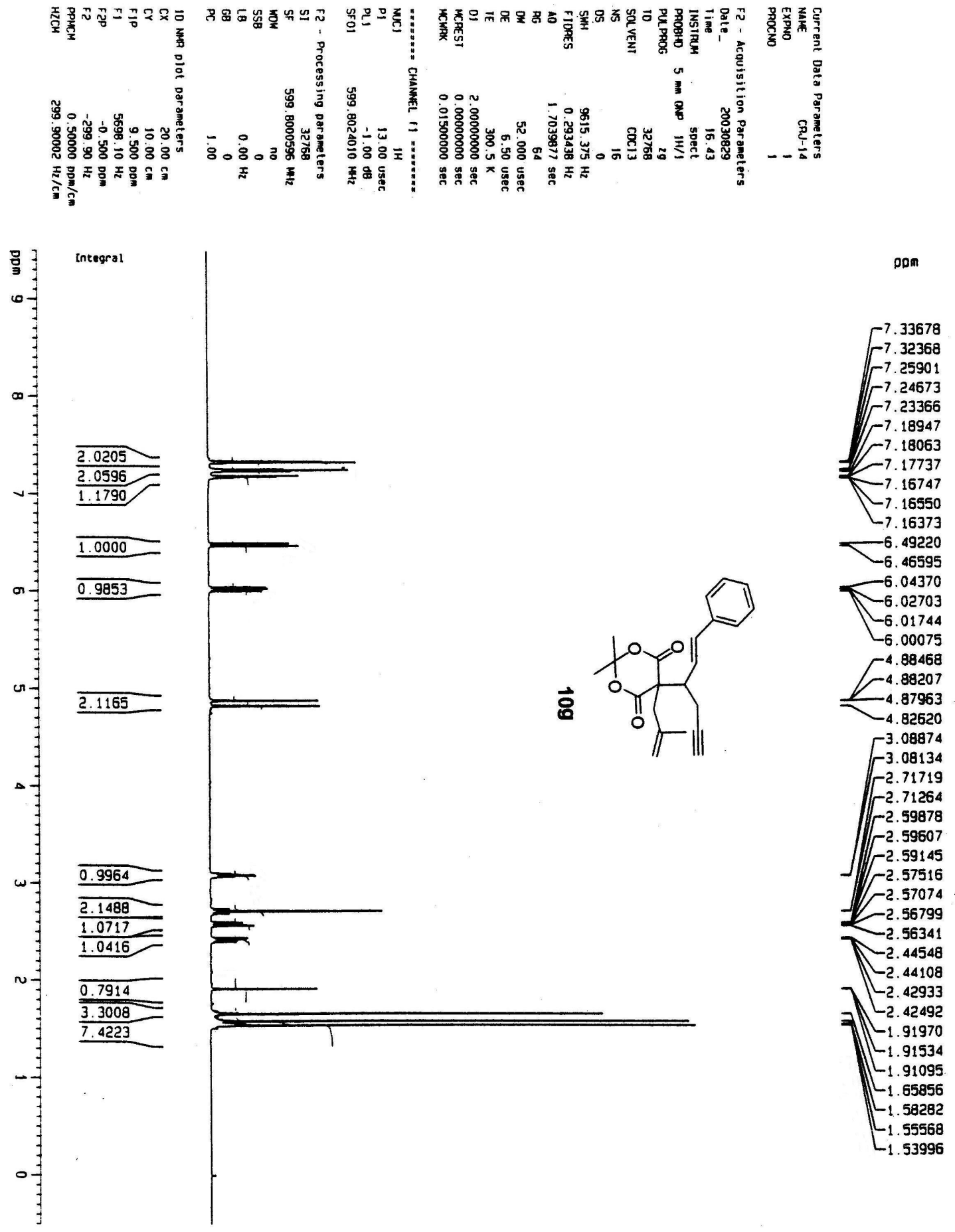

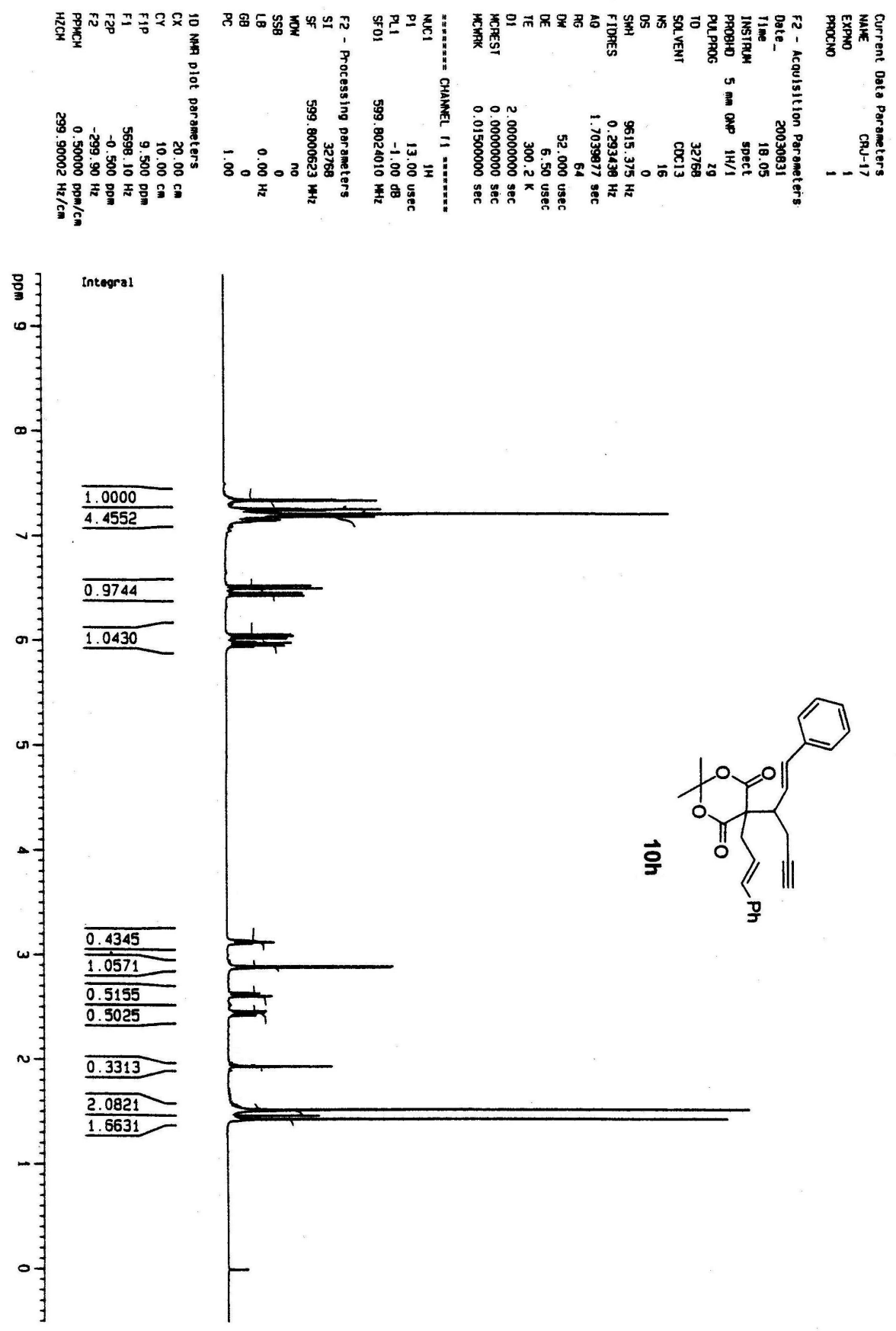

ppom

7.3511

$F^{7.3494}$

$\begin{array}{r}7.3371 \\ -7.2675 \\ \hline\end{array}$

7.257

$-7.2423$

7.2423
7.2138
7.2103

7.2138
7.2103

7.2042

$\begin{array}{r}7.2042 \\ -7.1995 \\ \hline\end{array}$

7.1977

- 7.1892

$-7.1848$

-7.1831
-6.5256

6.4994

- 6.4994

6.4310

6.0639

[ 6.0472

5.9800
-3.1313

$F^{3.1237}$

2.8932
2.8603

2.8803
2.6370

- 2.634

I 2.6296

$F^{2.6135}$

$-2.6060$

2.6015

-2.4682

$-2.4522$

$-2.4477$

2.4401

$\left[\begin{array}{l}2.4356 \\ -1.9391\end{array}\right.$

$\left[\begin{array}{l}1.9391 \\ 1.9346\end{array}\right.$

$\left[\begin{array}{l}1.9346 \\ 1.9302\end{array}\right.$

$-1.9302$

$\left[\begin{array}{l}1.5215 \\ 1.4638\end{array}\right.$

$\left[_{1.4318}\right.$ 